\title{
Sustainable and Reusable Sulfonic Acid-Functionalized Task-Specific Ionic Liquid Catalysts for Multicomponent Reactions. A Review
}

\author{
Nicolas S. dos Anjos ${ }^{\circledR a}$ and Luiz S. Longo Jr. ${ }^{\circledR} * a$ \\ ${ }^{a}$ Departamento de Ciências Farmacêuticas, Instituto de Ciências Ambientais, Químicas e Farmacêuticas, \\ Universidade Federal de São Paulo, Rua São Nicolau 210, 09913-030 Diadema-SP, Brazil
}

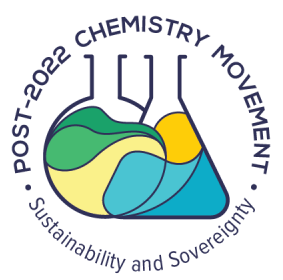

Improvements in Sustainable Organic Synthesis are usually related to the investigation of less hazardous, non-corrosive and renewable materials, as well as waste prevention, energy efficiency, solvent-free reactions, and the development of efficient reusable catalysts. In this context, multicomponent reactions emerged as important strategies for the rapid and more sustainable synthesis of organic molecules. In addition, the use of task-specific ionic liquids as non-conventional solvents and/or catalysts in multicomponent reactions has recently received great attention, contributing to the development of greener synthetic methodologies. This review describes the studies on the use of structurally diverse homogeneous and heterogeneous sulfonic acid-functionalized task-specific ionic liquids as acidic catalysts for multicomponent reactions applied to the synthesis of nitrogen- and oxygen-based heterocycles, as well as related compounds. The combination of the green credentials of multicomponent reactions and acidic ionic liquids are able to improve process sustainability and green metrics, contributing to sustainable chemical development and, ultimately, to economic growth and cleaner industrial production.

Keywords: acidic ionic liquids, Brønsted acidic ionic liquids, multicomponent reactions, reusable catalysts, sustainable organic synthesis

\section{Introduction}

Complex chemical manufacture processes allow us to rely on the availability of priceless products for modern life, such as new drugs to treat diseases and sophisticated materials for electronics (i.e., polymers or nanomaterials), among many others. However, these processes are often unsustainable in terms of resources, usually depending on non-renewable petroleum feedstocks. ${ }^{1}$ As important as the source of the starting materials for a given chemical process, the method itself has constantly challenged academic and industrial chemists to develop greener chemical production. Organic Chemistry has a pivotal role in defining new and greener methods for transformation of matter, taking into account the 12 Principles of Green Chemistry. Improvements in this field usually consist on the search for solvent-free reactions, the use of biomassderived solvents and/or starting materials, development of homogeneous or heterogeneous reusable catalysts as well as alternative heating methods, attempting to circumvent the

*e-mail: luiz.longo@unifesp.br

Editor handled this article: Luiz Ramos (Guest) drawbacks of classical procedures in Organic Synthesis. ${ }^{2}$ Moreover, the environmental importance of techniques such as mechanochemistry, flow chemistry, ultrasound and microwave-assisted heating have significantly improved small and large scale organic synthetic processes. ${ }^{3}$

In this scenario, multicomponent reactions (MCRs) emerged as powerful tools for efficient synthesis of organic compounds. This highly atom-efficient convergent strategy combines three or more starting materials in a one-pot reaction to afford a single product with several new chemical bonds being formed. ${ }^{4} \mathrm{Often}$, the multicomponent approaches benefit from being carried out under mild conditions (energy efficiency), in ordinary or nonconventional solvents, with the aid of homogeneous or heterogeneous catalysts, low by-product formation and easy purification procedures..$^{5-8}$ Indeed, the green credentials of multicomponent reactions are able to boost Chemistry for Sustainable Development, contributing to economic growth and cleaner industrial production. ${ }^{8}$

Ionic liquids (ILs) are versatile molten salts with melting point below $100{ }^{\circ} \mathrm{C}$, formed by an organic cation (e.g., imidazolium, benzimidazolium, pyridinium, pyrrolidinium, tetraalkylammonium, tetraalkylphosphonium, sulfonium, 
etc.) and halides or weak-coordinating anions, usually $[\mathrm{Cl}]^{-}$, $[\mathrm{Br}]^{-},\left[\mathrm{BF}_{4}\right]^{-},\left[\mathrm{PF}_{6}\right]^{-},\left[\mathrm{NTf}_{2}\right]^{-},\left[\mathrm{HSO}_{4}\right]^{-},[\mathrm{OTs}]^{-},[\mathrm{OTf}]^{-.}{ }^{9}$ In the past decades, ILs received great attention due to their attractive physical and chemical properties, such as low vapor pressure, non-inflammability, chemical and thermal stabilities, and high ionic conductivity, among others. ${ }^{10-13}$ Interestingly, some properties can be easily adjusted for a specific function, when cationic and anionic moieties are properly combined, the so-called task-specific ionic liquids (TSILs). ${ }^{14-17}$ For example, acidic ionic liquids (AILs) constitute an important class of TSILs with a wide range of applications. ${ }^{18,19}$ Recently, Amarasekara ${ }^{19}$ reviewed the role of Lewis and Brønsted acidic ionic liquids in chemistry, discussing their synthesis, physical and chemical properties as well as their applications in catalysis, analytical assays, biomass conversion, etc. More specifically, Brønsted acidic ionic liquids (BAILs) are proton donor compounds in which one or more acidic hydrogens are present in functional groups (i.e., $-\mathrm{SO}_{3} \mathrm{H}$ or $\mathrm{CO}_{2} \mathrm{H}$ ) attached to the cationic, anionic or both moieties. Recently, Song et al. ${ }^{20}$ reported experimental $\mathrm{pH}$ measurements and theoretical dissociation mechanism studies of $\mathrm{pH}$ values for some $\mathrm{SO}_{3} \mathrm{H}$ monofunctionalized ionic liquids. In general, BAIL acidity is anion-dependent, being higher for ionic liquids bearing acidic anions such as $\left[\mathrm{HSO}_{4}\right]^{-}$as well as for ionic liquids containing weak-coordinating anions, for instance [OTf $]^{-}$, [OTs $]^{-}$or $\left[\mathrm{CF}_{3} \mathrm{CO}_{2}\right]^{-}$. Furthermore, $\mathrm{SO}_{3} \mathrm{H}$-functionalized acidic ionic liquids display remarkable catalytic activities in many organic transformations. ${ }^{17}$

The role of ionic liquids as catalysts and/or solvents for organic reactions was extensively reviewed over the past two decades or so. ${ }^{10,12,21-23}$ In 2011, Isambert et al. ${ }^{21}$ described the synergic relationship between ionic liquids and multicomponent reactions applied to the synthesis of heterocyclic compounds, highlighting their sustainable credentials. In addition, immobilization of ionic liquids into a solid supporting material (SILP, supported ionic liquid phases) emerged as powerful strategy to reduce catalyst loadings and to facilitate product separation from reaction media. ${ }^{24-29}$ More specifically, heterogeneous supported Brønsted or Lewis acidic ionic liquid phases have been acknowledged as efficient catalysts for many organic reactions..$^{30}$ Ultimately, to boost process sustainability and green metrics, acidic ionic liquid-based systems offer key benefits for the development of advanced technologies, allowing the replacement of hazardous and corrosive strong acidic catalysts, as well as integrating catalytic efficiency/ activity, catalyst recovery and easy product separation. ${ }^{31}$

In this review contribution, we discuss and compare the studies on the use of structurally diverse homogeneous and heterogeneous sulfonic acid-functionalized task-specific ionic liquids as catalysts in several multicomponent reactions for the synthesis of nitrogen- and oxygen-based heterocycles, among others, as efficient and green methods for the development of sustainable chemical processes in Organic Synthesis.

\section{Multicomponent Reactions Catalyzed by $\mathrm{SO}_{3} \mathrm{H}$-Functionalized Ionic Liquids}

\subsection{Synthesis of dihydropyrimidinones}

Since the $19^{\text {th }}$ century, Biginelli multicomponent reaction has been considered the most efficient method to prepare 3,4-dihydropyrimidin-2(1H)-(thi)ones (DHPM), ${ }^{32}$ an important class of heterocycles with broad spectrum of biological activities. ${ }^{33}$ The conventional methods for Biginelli MCR may impose some drawbacks such as the need of strong acidic conditions, low yields and poor stereoselective control. ${ }^{34}$ Consequently, the optimization of reaction conditions has been studied in order to find cheaper and more efficient catalysts, as well as milder and greener reaction conditions for the synthesis of DHPM derivatives and Biginelli asymmetric approaches. ${ }^{35-38}$

Task-specific ionic liquids containing $\mathrm{SO}_{3} \mathrm{H}-$ functionalized groups can act as efficient Brønsted acidic catalysts in Biginelli multicomponent reactions. For example, Abbasi ${ }^{39}$ investigated the use of 1,3-disulfonic acid benzimidazolium chloride $\mathbf{1}$ as catalyst in Biginelli one-pot condensation of aromatic aldehydes, ethyl acetoacetate and urea/thiourea (Scheme 1). The corresponding DHPMs were obtained in excellent yields (88-97\%) and shorter reaction time, using only $10 \mathrm{~mol} \%$ of $\mathbf{1}$ at $80{ }^{\circ} \mathrm{C}$ under solventfree conditions. The recyclability of the catalyst was also studied, and the catalyst could be reused in five consecutive cycles without a noticeable decrease in the yields (90-95\%). In a very similar study, Zare and Nasouri ${ }^{40}$ showed that $N, N$-diethyl- $N$-sulfoethanaminium hydrogen sulfate, $\left[\mathrm{Et}_{3} \mathrm{~N}-\mathrm{SO}_{3} \mathrm{H}\right]\left[\mathrm{HSO}_{4}\right]$, can also act as efficient Brønsted acidic catalyst for Biginelli MCR. In another approach, Savanur et al. ${ }^{41}$ studied the catalytic systems composed of imidazolium-based ionic liquids, $\left[\mathrm{C}_{4} \mathrm{C}_{1} \mathrm{Im}\right][\mathrm{X}]$, where $\mathrm{X}=\left[\mathrm{BF}_{4}\right]^{-}$or $\left[\mathrm{PF}_{6}\right]^{-}$, along with Brønsted acidic $\left[\left(\mathrm{SO}_{3} \mathrm{H}\right)^{4} \mathrm{C}_{4} \mathrm{C}_{1} \mathrm{Im}\right][\mathrm{OTf}]$ and Lewis acidic $\mathrm{Zn}\left(\mathrm{NTf}_{2}\right)_{2}$ as non-conventional media for Biginelli MCR. Finally, the microwave-assisted synthesis of DHPMs via Biginelli reaction was also studied by Wang et al. ${ }^{42}$ who employed a dual functional $\mathrm{SO}_{3} \mathrm{H}$-functionalized poly(ethylene glycol) (PEG)-catalyst and solvent.

More recently, Braga et $a l .{ }^{43}$ reported a microwaveassisted approach for Biginelli MCR catalyzed by $\mathrm{SO}_{3} \mathrm{H}$-functionalized imidazolium-based ionic liquid $\mathbf{2}$, 


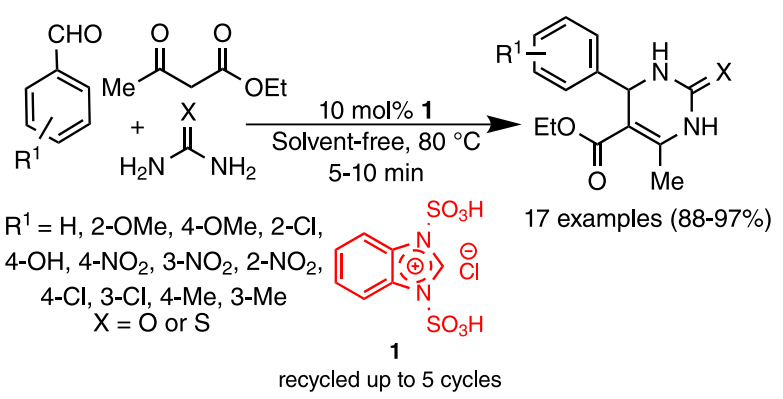

Scheme 1. Biginelli MCR catalyzed by 1,3-dissulfonic acid benzimidazolium chloride $\mathbf{1}$.

$\left[\left(\mathrm{SO}_{3} \mathrm{H}\right)^{4} \mathrm{C}_{4} \mathrm{C}_{4} \mathrm{Im}\right][\mathrm{Cl}]$, aiming at the synthesis of several dihydropyrimidin(thi)one derivatives as potential urease inhibitors. The authors studied the solvent-free Biginelli one pot condensation of ethyl acetoacetate, substituted aromatic aldehydes and urea/thiourea using $20 \mathrm{~mol} \%$ of 2 under microwave-assisted heating at $90{ }^{\circ} \mathrm{C}$ for $10 \mathrm{~min}$, which gave the corresponding DHPMs in variable yields (4-92\%). The lowest yield (ca. 4\%) was obtained when aliphatic cyclohexanecarboxaldehyde $\left(\mathrm{R}^{1}=c \mathrm{C}_{6} \mathrm{H}_{11}\right)$ was used as the carbonyl component (Scheme 2).

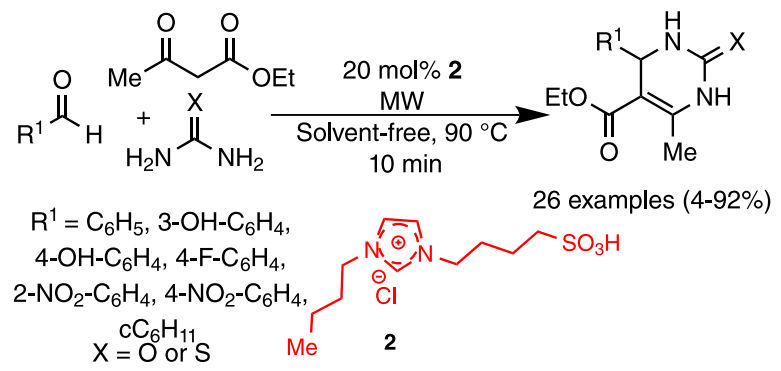

Scheme 2. Microwave-assisted Biginelli reaction catalyzed by 2 .

Rahman et al. ${ }^{44}$ synthesized benzoquinazoline derivatives via modified Biginelli approach using acidic ionic liquids based on 1-methyl-3-sulfoalkyl-imidazolium cation. The catalytic performance was evaluated in a solvent-free model reaction between $\alpha$-tetralone (enolizable carbonyl component), benzaldehyde and urea, with only $1 \mathrm{~mol} \%$ of the acidic catalyst. The generality and scope of this protocol were successfully studied for the preparation of several benzoquinazoline derivatives in good to high yields (68-85\%), when 1-methyl-3-(4-sulfobutyl)imidazolium 4-methylbenzenesulfonate $3,\left[\left(\mathrm{SO}_{3} \mathrm{H}\right)^{4} \mathrm{C}_{4} \mathrm{C}_{1} \mathrm{Im}\right][\mathrm{OTs}]$, was used as catalyst (Scheme 3 ).

Long alkyl chain BAILs have also been evaluated as catalysts for Biginelli MCR. In a comparative study, Zhou et al. ${ }^{45}$ screened the catalytic performance of several $\mathrm{SO}_{3} \mathrm{H}$-functionalized 1,3-dialkyl-imidazolium catalysts for this reaction, using cyclopentanone as enolizable carbonyl component, urea and two equivalents of substituted aromatic aldehydes (Scheme 4). The best

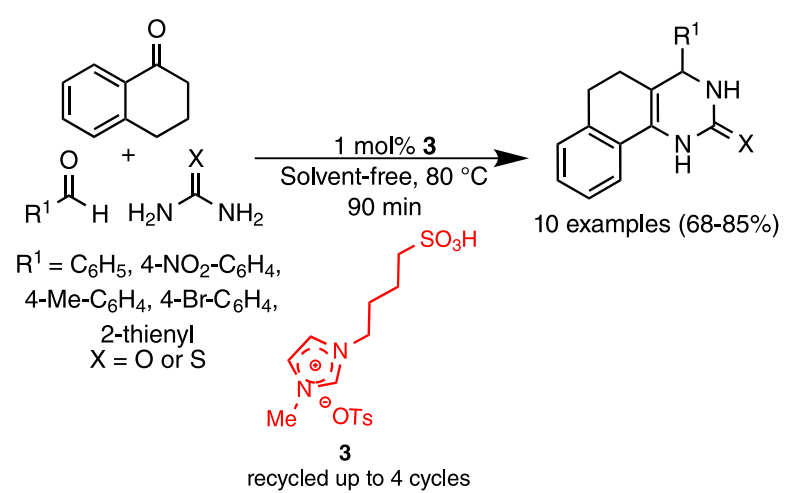

Scheme 3. Preparation of benzoquinazoline derivatives through modified Biginelli reaction catalyzed by 3 .

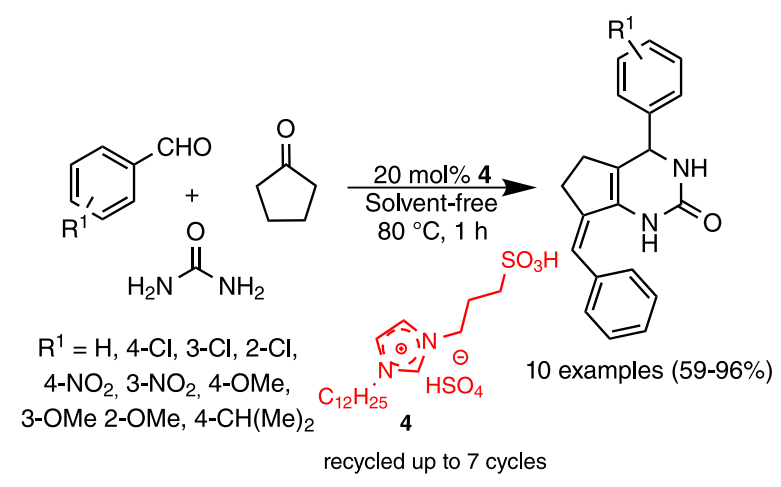

Scheme 4. Biginelli-type reaction catalyzed by $\mathrm{SO}_{3} \mathrm{H}$-functionalized 1,3-dialkylimidazolium catalyst 4.

catalyst was 1-dodecyl-3-(3-sulfopropyl)-imidazolium hydrogen sulfate $4,\left[\left(\mathrm{SO}_{3} \mathrm{H}\right)^{3} \mathrm{C}_{3} \mathrm{C}_{12} \mathrm{Im}\right]\left[\mathrm{HSO}_{4}\right]$, used in $20 \mathrm{~mol} \%$ under solventless conditions at $80^{\circ} \mathrm{C}$, furnishing the desired DHPMs in high yields (59-96\%). According to the authors, the long alkyl chain attached to the $\mathrm{N} 1$ of imidazolium cation improves catalyst solubility in organic phase and therefore enhances the interactions between catalyst and reactants. The catalyst $\mathbf{4}$ could be recycled and reused up to seven times without significant loss in catalytic activity. In a similar approach, He et al. ${ }^{46}$ also demonstrated the catalytic efficiency of a series of longchain $\mathrm{SO}_{3} \mathrm{H}$-functionalized tetraalkyl ammonium ionic liquids for Biginelli multicomponent reaction.

Recently, Alvim et al..$^{47}$ combined an asymmetric counter anion-directed catalysis (ACDC) approach with ionic liquid effects in the enantioselective Biginelli MCR for the synthesis of chiral DHPMs. The three-component reaction proceeded smoothly with $10 \mathrm{~mol} \%$ of chiral catalyst 5 , in neat conditions at $30^{\circ} \mathrm{C}$ for three days, furnishing the desired DHMPs in good to excellent yields and variable enantiomeric excess (ee) values (Scheme 5). This strategy relies on both, chiral induction and Brønsted acid catalysis of chiral ionic liquids. The observed enantioselectivities could be explained by the unprecedented formation of supramolecular aggregates (interaction between the key 


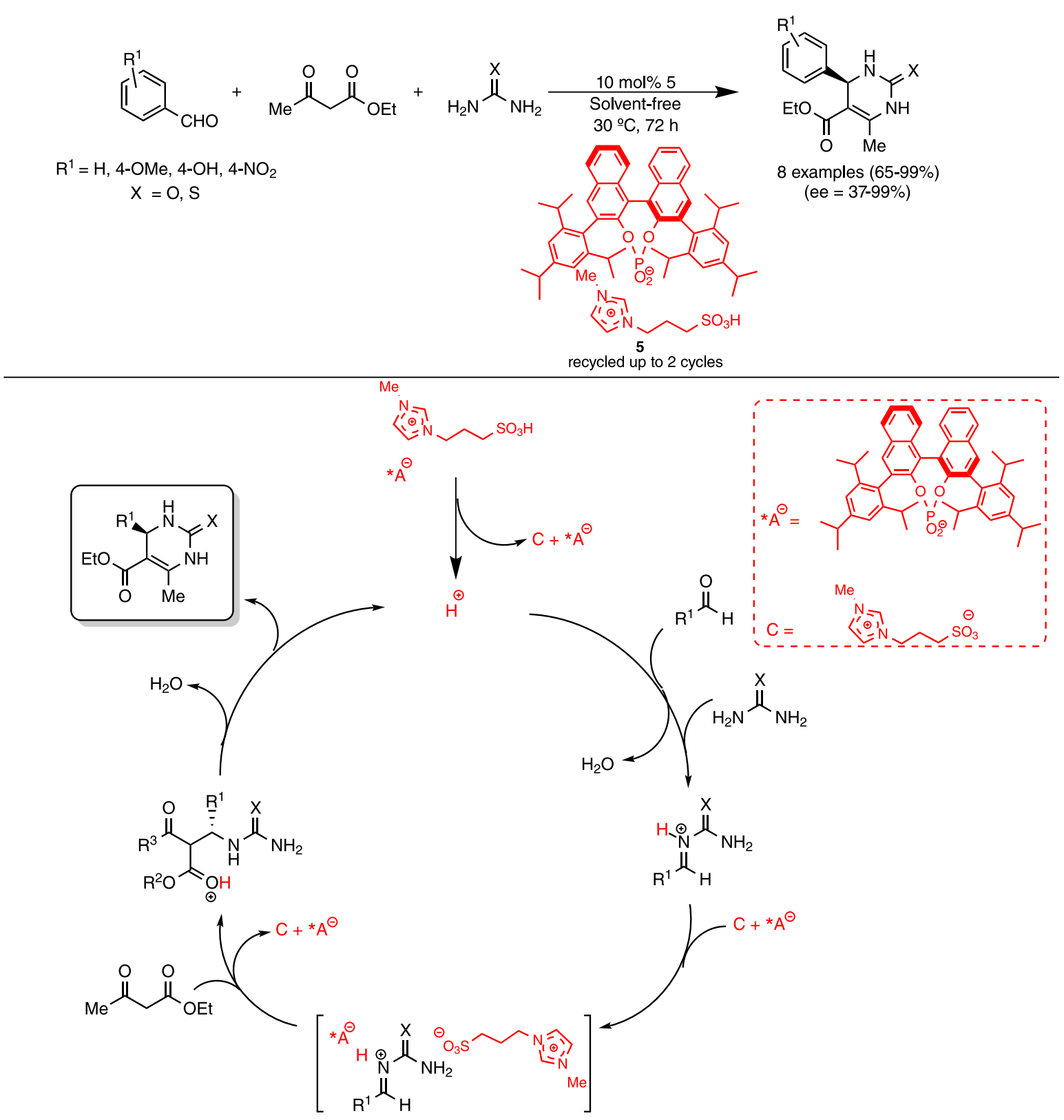

Scheme 5. Enantioselective Biginelli MCR catalyzed by dual-functional BAIL 5.

intermediate iminium cation and the chiral ionic liquid), demonstrated by electrospray tandem mass spectrometry experiments. Theoretical calculations of transition state energies showed that the mechanism pathway involves the formation of iminium intermediates (attack of urea to the protonated aldehyde), and the chiral induction is a result of the favored si face attack by the 1,3-dicarbonyl starting material to the iminium intermediate, leading to $(R)$-DHPMs as major isomers.

Heteropolyanion-based ionic liquids (HPAILs) were also investigated as catalysts for Biginelli multicomponent reactions. ${ }^{48,49}$ This class of acidic ionic liquid possesses interesting physicochemical properties such as high melting point as well as high thermal and chemical stabilities, which facilitate catalyst recovering from reaction media. In the study reported by Fu et al., ${ }^{48}$ the catalysts 6, 7 and $\mathbf{8}$, bearing different $\mathrm{SO}_{3} \mathrm{H}$-decorated cationic heads, showed high catalytic efficiency in solvent-free Biginelli reactions between urea, ethyl acetoacetate and different aldehydes under microwave heating (Scheme 6). According to the authors, one of the major advantages of the HPAILs is their immiscibility with some esters (i.e., ethyl acetate), facilitating recyclability of the catalyst and making the procedure more sustainable. Therefore, the catalyst 8 could be recovered from the reaction mixture upon simple filtration after stirring with hot ethyl acetate, allowing it to 
be reused up to five reaction cycles without loss of catalytic performance.

Another eco-friendly catalytic approach for organic transformations relies on the use of supported ionic liquid phases (SILPs) in which the $\mathrm{SO}_{3} \mathrm{H}$-funcionalized ionic liquid moiety is covalently attached to a solid matrix, usually polymers, nanoparticles, carbon nanotubes or silicabased materials. ${ }^{24,25}$ As solid heterogeneous catalysts, they can be easily recovered by simple filtration techniques, often improving recyclability, and consequently process green metrics. ${ }^{50}$ Khiratkar et al..$^{51}$ studied the Biginelli MCR catalyzed by poly(vinylbenzyl) polymer-supported $\mathrm{SO}_{3} \mathrm{H}$-decorated benzimidazolium-based ionic liquids (Scheme 7). The heterogeneous catalyst 9 was synthesized starting from 4-vinylbenzyl chloride, benzimidazole and 1,4-butane sultone, and it was further evaluated for a model reaction between urea, benzaldehyde and ethyl acetoacetate. The best results were observed when the reaction was carried out in the presence of $50 \mathrm{mg} \mathrm{mmol}^{-1}$ of 9 , using ethanol as solvent at $110^{\circ} \mathrm{C}$ for $18 \mathrm{~h}$. A total of twenty-four DHPMs were obtained in moderate to excellent yields, depending on the nature of the aldehyde component; electron-rich aryl aldehydes led to DHPMs in higher yields whereas electron-poor aryl aldehydes and aliphatic aldehydes furnished the desired products in lower yields. The recyclability and stability of the catalyst were also studied in consecutive Biginelli reactions, and $\mathbf{9}$ could be recovered and reused up to five reaction cycles without significant loss in catalytic efficiency. Rostamizadeh et al..$^{52}$ also prepared a heterogeneous Brønsted acidic ionic liquid catalyst for Biginelli-type synthesis of pyrimido[4,5- $d]$ pyrimidine derivatives. The nanocatalyst 10, containing an alkyl sulfonic acid ionic liquid immobilized on $\alpha-\mathrm{Fe}_{2} \mathrm{O}_{3}$-functionalized mesoporous silica (MCM-41), showed synergic catalytic effects due to the high surface area of $\alpha-\mathrm{Fe}_{2} \mathrm{O}_{3}-\mathrm{MCM}-41$ nanoparticles (containing $\mathrm{Fe}^{3+}$ species as Lewis acid) and the presence of $-\mathrm{SO}_{3} \mathrm{H}$ groups and $\left[\mathrm{HSO}_{4}\right]^{-}$anion as Brønsted acidic sites. Thus, a catalyst loading of
$60 \mathrm{mg} \mathrm{mmol}{ }^{-1}$ of $\mathbf{1 0}$ was able to efficiently catalyze the reaction of 6-aminouracil (as enolizable carbonyl equivalent), aldehydes and urea/thiourea, furnishing several pyrimido[4,5- $d$ ] pyrimidine derivatives in good to excellent yields (Scheme 7). After each reaction experiment, the heterogenous catalyst could be easily collected using an external magnet and reused up to six times with only a small decrease in catalytic activity (ca. 8\%). More recently, Yao et $a l .{ }^{53}$ successfully described the use of copper-doped MCM-41 supported acid-functionalized ionic liquid as an efficient and recyclable heterogeneous catalyst for Biginelli multicomponent reaction.

\subsection{Synthesis of 1,4-dihydropyridines}

The 1,4-dihydropyridines (1,4-DHP) represent another important class of nitrogen-based heterocycles with many applications in modern Organic and Medicinal Chemistry. ${ }^{54-59}$ The Hantzsch multicomponent reaction was firstly described in $1882,{ }^{60}$ and today it is widely acknowledged as an efficient method to prepare 1,4-DHPs by mixing together two equivalents of 1,3-dicarbonyl compounds (or a $\mathrm{CH}_{2}$-acid equivalent component), an aromatic aldehyde, and ammonia (usually as ammonium acetate) or a primary amine.

Several examples of Hantzsch multicomponent reaction to access 1,4-dihydropyridines and related compounds, using ordinary homogeneous Brønsted acidic ionic liquids as catalysts, have been described in the recent literature. ${ }^{61-63}$ Jassem et al. ${ }^{61}$ exploited a four-component version of Hantzsch reaction catalyzed by 3-methyl-1-sulfonic acid imidazolium chloride 11. The transformation between 4-hydroxybenzaldehyde, acetylacetone, several primary amines and barbituric acid (as 1,3-dicarbonyl equivalent), catalyzed by $10 \mathrm{~mol} \%$ of 11 using ethanol at room temperature, furnished the corresponding pyrido[2,3- $d$ ] pyrimidine$2,4(1 H, 3 H, 5 H, 8 H)$-dione derivatives in good to excellent yields (80-93\%) (Scheme 8 ).

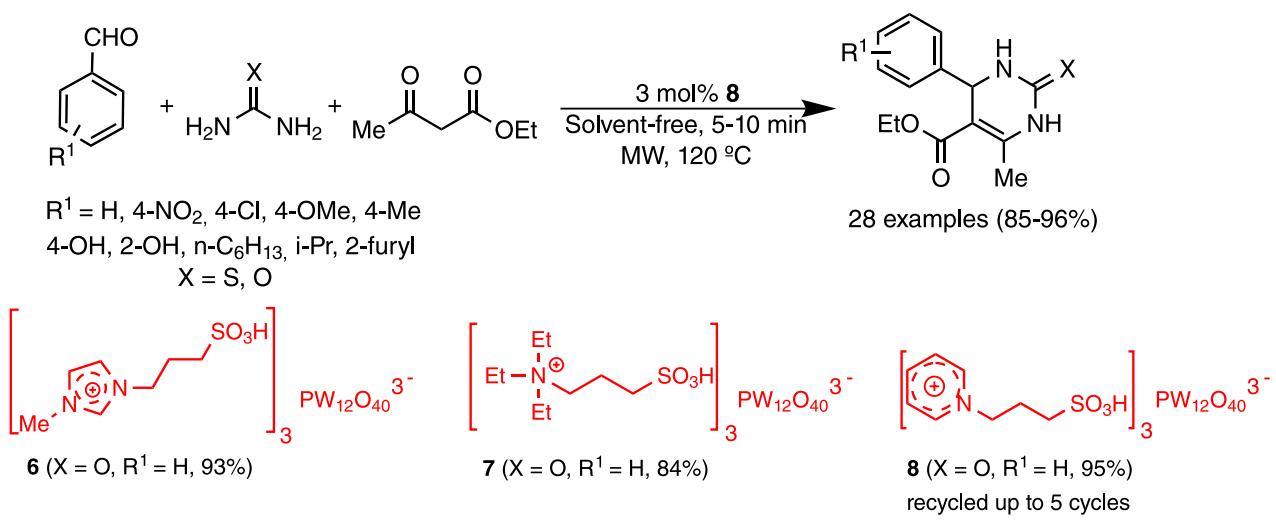

Scheme 6. HPAILs-catalyzed Biginelli multicomponent reaction. 


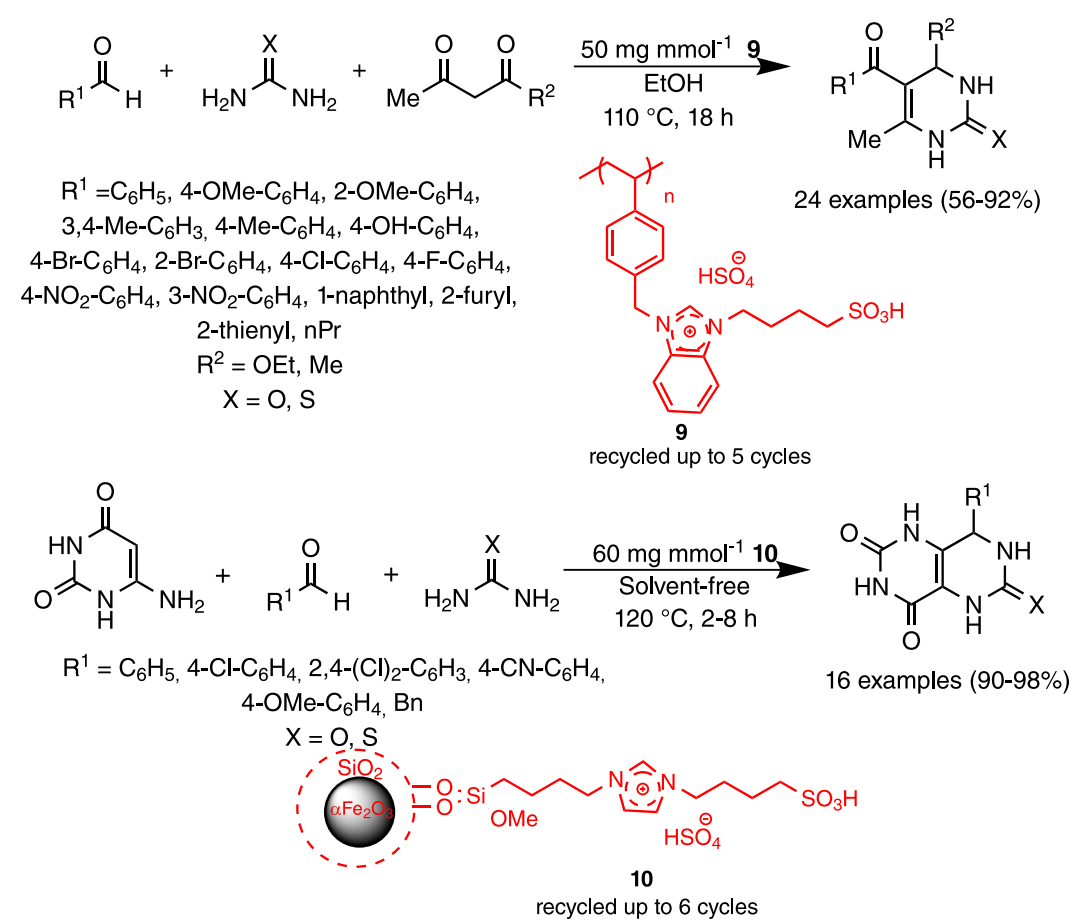

Scheme 7. Biginelli-like multicomponent reactions catalyzed by heterogeneous $\mathrm{SO}_{3} \mathrm{H}$-functionalized ionic liquid catalysts $\mathbf{9}$ and $\mathbf{1 0}$.

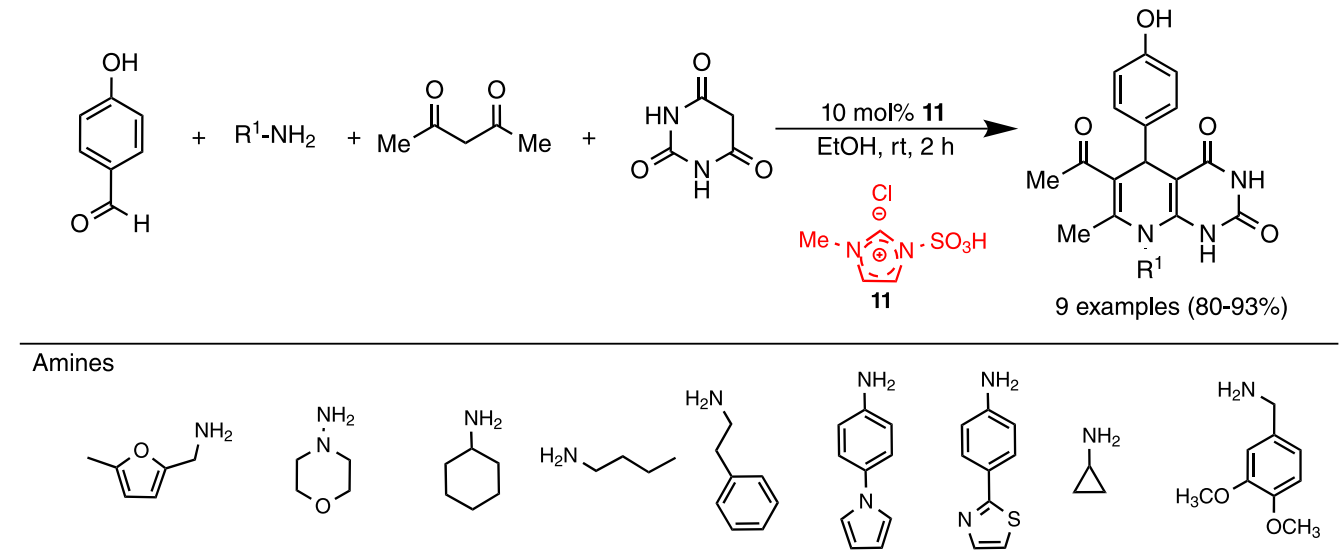

Scheme 8. Synthesis of 1,4-DHP derivatives via Brønsted acidic ionic liquid-catalyzed four-component Hantzsch reaction.

Liu and $\mathrm{Liu}^{62}$ evaluated the dual role of long alkyl chain $\mathrm{SO}_{3} \mathrm{H}$-funcionalized BAIL as catalyst and solvent for the one-pot tandem oxidation process (TOP) followed by Hantzsch multicomponent reaction. In this approach, the benzylic alcohol is oxidated in situ to the correspondent aldehyde, upon treatment with 40 mol\% of catalyst $\mathbf{1 2}$ and $\mathrm{NaNO}_{3}$, followed by the Hantzsch reaction with ammonium acetate and 1,3-ketoesters (Scheme 9). The 1,4-DHPs

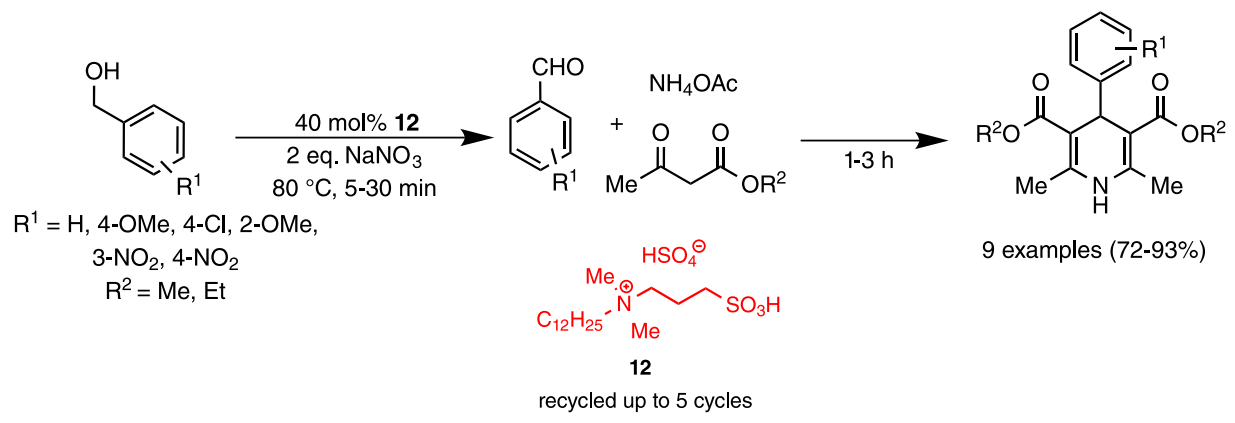

Scheme 9. Synthesis of 1,4-DHP through one-pot sequential oxidation/Hantzsch reaction catalyzed by $\mathbf{1 2}$ and $\mathrm{NaNO}_{3}$. 
were then obtained in good to excellent yields, depending on the substituent of the benzylic alcohol. In general, electron-rich aromatic alcohols react rapidly, leading to the corresponding products in higher yields, whereas electrondeficient aromatic alcohols tend to require longer reaction times, consequently resulting in lower product yields.

In 2012, Jahanbin et al. ${ }^{64}$ firstly studied the Hantzsch MCR catalyzed by polymer-supported $\mathrm{SO}_{3} \mathrm{H}$-functionalized ionic liquids as heterogenous catalysts. The authors used polystyrene-immobilized 3-methyl-1-(4-sulfonylbutyl) imidazolium hydrogen sulfate $\mathbf{1 3}$ as reusable catalyst for the reaction of ethyl acetoacetate, ammonium acetate and aromatic aldehydes in refluxing ethanol for few hours, furnishing the corresponding 1,4-dihydropyridines in excellent yields (Scheme 10). After each reaction run, the heterogeneous catalyst could be isolated and reused up to five consecutive cycles. In a similar study, Alvim et al. ${ }^{65}$ reported the role of catalyst 6 as HPAIL for the Hantzsch reaction using 1,3-dialkylimidazoliumbased ionic liquids, 1-butyl-3-methylimidazolium bis(trifluoromethylsulfonyl)imide, $\left[\mathrm{C}_{4} \mathrm{C}_{1} \mathrm{Im}\right]\left[\mathrm{NTf}_{2}\right]$, as nonconventional media (Scheme 10). The reaction between aromatic aldehydes (or formaldehyde), ammonium acetate and 1,3-dicarbonyl compounds, carried out in the presence of $5 \mathrm{~mol} \%$ of 6 immobilized in $\left[\mathrm{C}_{4} \mathrm{C}_{1} \mathrm{Im}\right]\left[\mathrm{NTf}_{2}\right]$ at $90{ }^{\circ} \mathrm{C}$ for $4 \mathrm{~h}$, afforded the 1,4-DHPs in good to excellent yields. Interestingly, the authors successfully used this protocol for the synthesis of $\beta$-amino carbonyl compounds (via Mannich MCR) and, more recently, for the synthesis of hexahydroimidazo[1,2-a]pyridines derivatives. ${ }^{65,66} \mathrm{In}$ addition, carefully planned electrospray ionization tandem mass spectrometry (ESI-MS/MS) experiments shed some light on the reaction mechanism, evidencing the origin of the ionic liquid effect as well as suggesting the formation of charged intermediates and supramolecular aggregates, which allowed the postulation of two concurrent reaction pathways leading to the same final adduct.

Ren et $a l .{ }^{67}$ firstly described a class of poly(ethylene glycol)-linked dicationic acidic ionic liquids (PEG-DAILs) as catalyst for the Hantzsch reaction. A biphasic system composed of $\mathrm{PEG}_{1000}$-DAIL/toluene (2:3) was studied, showing temperature-dependent phase behavior (monophasic at higher temperatures and biphasic at lower temperatures), which facilitates the recyclability of the ionic liquid. This unconventional media system was used in the reaction of electronrich and electron-deficient aromatic aldehydes, ethyl acetoacetate and ammonium acetate, at $80{ }^{\circ} \mathrm{C}$ for $40 \mathrm{~min}$, furnishing the 1,4-DHPs in good to excellent yields (86-96\%) (Scheme 11). The authors studied the recovery of this heterogenous catalyst using a Hantzschlike multicomponent reaction between benzaldehyde, 1,3-cyclohexanedione, ethyl acetoacetate and ammonium acetate, which furnished ethyl 1,4,5,6,7,8-hexahydro2-methyl-5-oxo-4-phenylquinoline-3-carboxylate in high yield (> 90\%) (see section 2.3). Upon cooling, the $\mathrm{PEG}_{1000}$-DAIL/toluene system became biphasic and the bottom layer containing the $\mathrm{PEG}_{1000}$-DAIL catalyst could be easily separated and reused up to nine cycles without any special treatment. Later, the research group
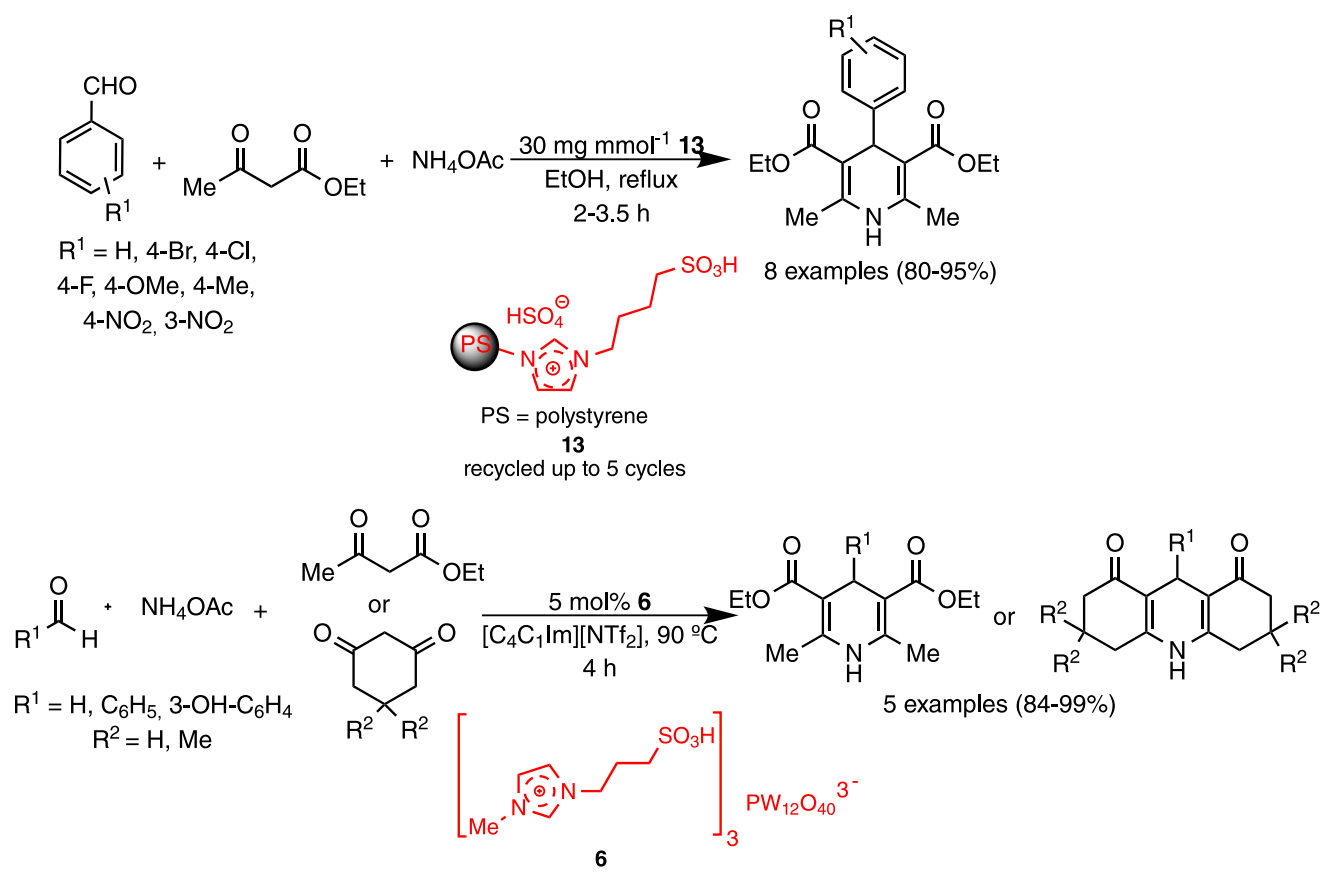

Scheme 10. Synthesis of 1,4-dihydropyridines via Hantzsch MCR catalyzed by polymer-supported catalyst 13 and HPAIL 6. 
applied the same protocol to the synthesis of complex nitrogen-based heterocycles, such as 4-aryl-3-methyl1 -phenyl-1 $H$-benzo[ $h]$ pyrazolo $[3,4-b]$ quinoline5,10-diones, 3-arylbenzoquinolines, pyranoquinolines and thiopyranoquinoline derivatives. ${ }^{68,69}$

Triazolium-based Brønsted acidic ionic liquids supported on iron-containing MCM-41 nanoparticles were synthesized and characterized by Naeimi et al. ${ }^{70}$ The authors exploited these heterogeneous catalysts in a Hantzsch-like multicomponent reaction in aqueous media for the synthesis of tricyclic nitrogen-based heterocycles. Surface acidity of Fe ${ }^{\mathrm{III}}$-MCM-41-IL $\mathbf{1 5}$ was measured by $\mathrm{NaOH}$ titration ( $\mathrm{pH}$ value was 1.04 ), corresponding to a loading of $2.19 \mathrm{mmol}\left(\mathrm{H}^{+}\right) \mathrm{g}^{-1}$. A model reaction between benzaldehyde, ammonium acetate and 2 equivalents of 2-thiobarbituric acid (as 1,3-dicarbonyl component) was selected to establish the scope and limitations of the method. Using the optimized conditions, the reactions proceeded smoothly with catalyst $\mathbf{1 5}$ in water at room temperature, affording the desired products in good to excellent yields (Scheme 12). After each reaction cycle, the catalyst was recovered by attaching an external magnet to the reaction flask, followed by washing with hot water and drying under vacuum. The catalyst could then be reused up to six cycles with constant catalytic activity (yields vary from 95 to $93 \%$ in all cycles).

More recently, Mirhosseini-Eshkevari et al. ${ }^{71}$ prepared $\mathrm{SO}_{3} \mathrm{H}$-functionalized ionic liquids supported on zirconiumbased metal-organic frameworks (MOFs) as nanocatalyst for the one-pot synthesis of dihydropyrido[2,3- $d$ ]pyrimidine derivatives. The crystalline porous heterogenous catalyst was composed of metal ions and organic linkers decorated with Brønsted acidic ionic liquids, BAIL@UiO-66 16, combining the MOF nanocages physical and chemical properties with ionic liquid Brønsted acidity, resulting in a greater amount of acidic catalytic active sites and excellent catalytic performance. The catalytic efficiency of $\mathbf{1 6}$ was demonstrated in the multicomponent reaction between arylaldehydes, acetylacetone and 6-amino1,3-dimethyl uracil as 1,3-dicarbonyl equivalent, leading to dihydropyrido $[2,3-d]$ pyrimidine derivatives in excellent yields (usually $>90 \%$ ) (Scheme 13). The green credentials of the method rely on the use of solventless conditions, short reaction time, clean work-up procedure as well as easy catalyst recycling (reused up to six cycles without loss in catalytic efficiency).

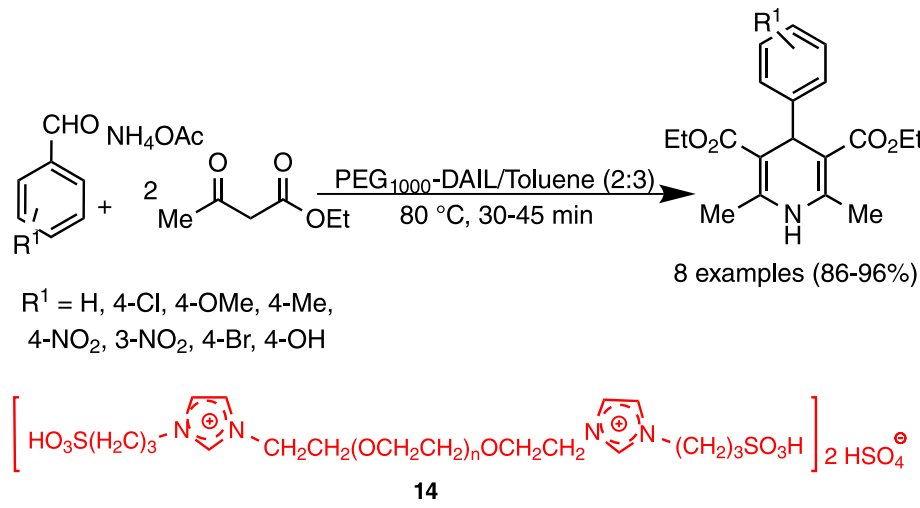

Scheme 11. Synthesis of 1,4-DHPs catalyzed by PEG $_{1000}$-DAIL 14.

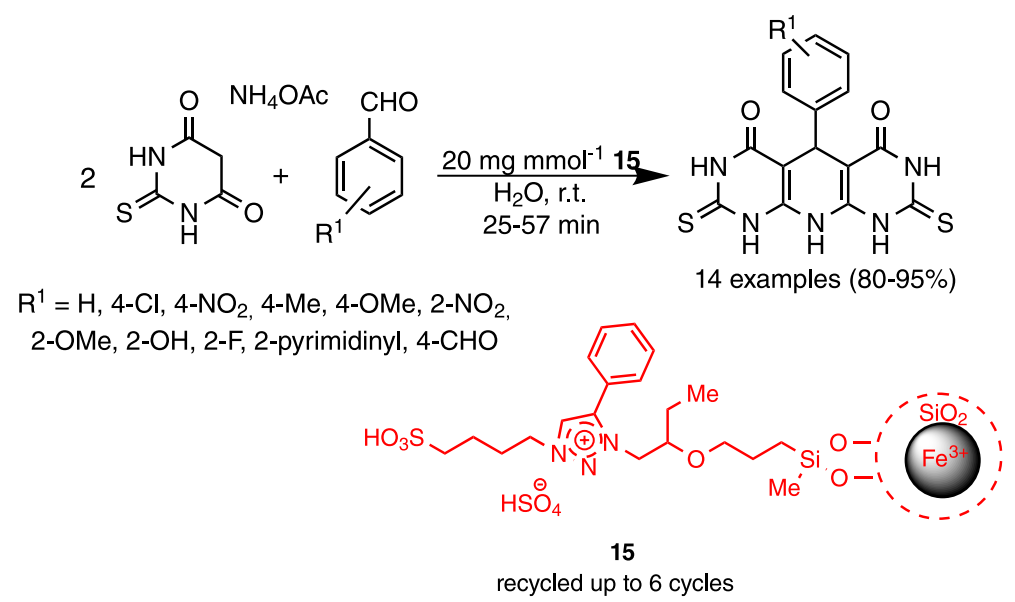

Scheme 12. Synthesis of pyrimidine derivatives through four-component synthesis catalyzed by BAIL immobilized on Fe ${ }^{\mathrm{III}}-\mathrm{MCM}-41-\mathrm{IL}$ 15. 
<smiles>CC(=O)CC(C)=O</smiles>
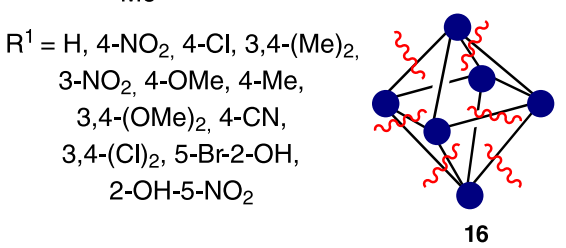

12 examples $(90-98 \%)$

recycled up to 6 cycles
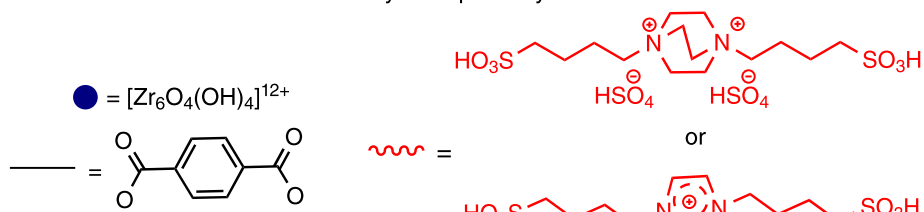

$\sim m=$

or

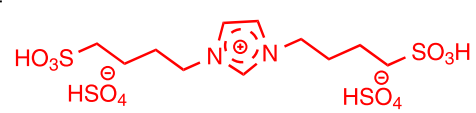

Scheme 13. Synthesis of dihydropyrido[2,3- $d$ ]pyrimidine derivatives through MCR catalyzed by MOF BAIL@UiO-66 16.

A general mechanism for the Hantzsch reaction catalyzed by $\mathrm{SO}_{3} \mathrm{H}$-functionalized BAILs is depicted in Scheme 14 (exemplified for symmetrical substituted 1,4-dihydropyridines). Brønsted acid catalysis is involved in the initial formation of Knoevenagel adduct $\mathbf{I}$ and enamine II. Then, Michael addition of nucleophilic enamine II to the $\alpha, \beta$-unsaturated carbonyl compound $\mathbf{I}$ leads to the intermediate III. The later undergoes an intramolecular condensation of the amino and carbonyl groups catalyzed by $\mathrm{SO}_{3} \mathrm{H}$-functionalized BAILs to furnish IV, which in turn leads to 1,4-dihydropyridines after abstraction of a proton.<smiles>[R]OC(=O)CC(C)=O</smiles>

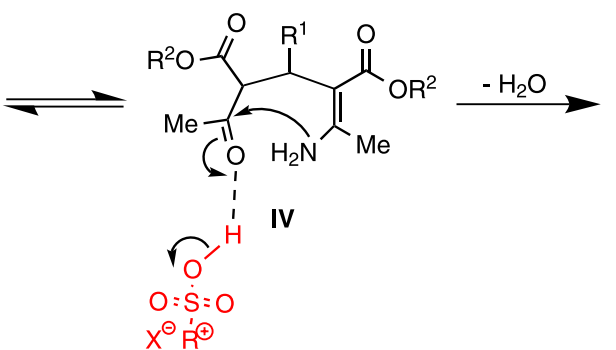

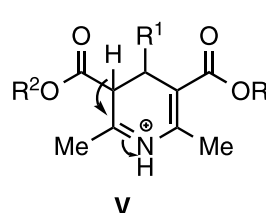

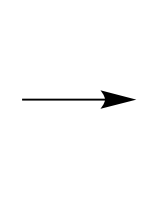<smiles></smiles>

1,4-DHPs

Scheme 14. Reaction mechanism for the Hantzsch reaction catalyzed by $\mathrm{SO}_{3} \mathrm{H}$-functionalized BAILs. 


\subsection{Synthesis of quinoline derivatives and related} compounds

Nitrogen-based heterocycles containing the quinoline moiety are very important in Medicinal Chemistry because of their wide range of pharmacological properties such as antimalarial, antitumor and antitubercular activities, among others. ${ }^{72-75}$ Quinoline derivatives (natural products, alkaloids and synthetic quinoline libraries) can be synthesized by different methods, usually requiring expensive starting materials, hazardous solvents and/or high temperature conditions. Recently, Batista et al. ${ }^{75}$ discussed the green methods and metrics for the synthesis of quinoline compounds employing more efficient energy methods with less hazardous solvents as well as the use of renewable and eco-friendly catalysts.

Many studies describing the synthesis of quinoline derivatives, i.e., benzo[ $g]$ indeno[2, $1-b]$ quinoline, pyrimido[4,5- $b$ ]quinolines or hexahydroquinolines, using multicomponent reactions catalyzed by $\mathrm{SO}_{3} \mathrm{H}$-functionalized BAILs were reported in the literature. Often these protocols rely on rapid, green and efficient synthetic methodologies, using acidic ionic liquids with the sulfonic acid group directly attached to the imidazolium cation. For instance, Zhang et $a l .{ }^{76}$ firstly described the use of $\mathbf{1 7}$ as a simple, cheap and reusable Brønsted acidic ionic liquid catalyst for pseudo three-component solvent-free reactions between two equivalents of enolizable aldehydes and substituted anilines. The reaction was carried out with $15 \mathrm{~mol} \%$ catalyst loading at $80{ }^{\circ} \mathrm{C}$ during 3-5 h, furnishing the expected 2,3,6-trisubstituted quinolines in moderate to high yields (51-86\%), along with the mono-alkylated and di-alkylated arylamines as side products (Scheme 15).

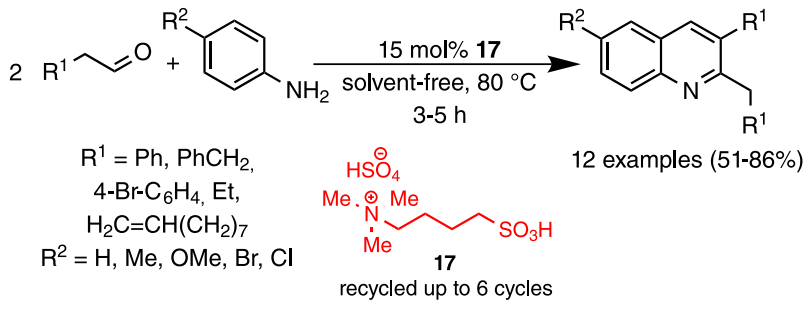

Scheme 15. Synthesis of 2-alkylquinolines catalyzed by BAIL 17.

In 2014, Khaligh ${ }^{77}$ described the synthesis of 12-arylbenzo $[g]$ indeno[2,1- $b]$ quinoline-6,11,13-triones using multicomponent reactions between substituted benzaldehydes, 2-hydroxynaphtalene-1,4-dione, $2 \mathrm{H}$-indene-1,3-dione and ammonium acetate catalyzed by acidic ionic liquid 18. A similar catalyst (19) was used by Mohammadi et al. ${ }^{78}$ in the one-pot multicomponent approach for the synthesis of pyrimido[4,5-b]quinoline derivatives. Both nitrogen-linked sulfonic acid catalysts could be easily recovered from the reaction media and reused up to 3 or 5 cycles without significant loss in activity (Scheme 16).
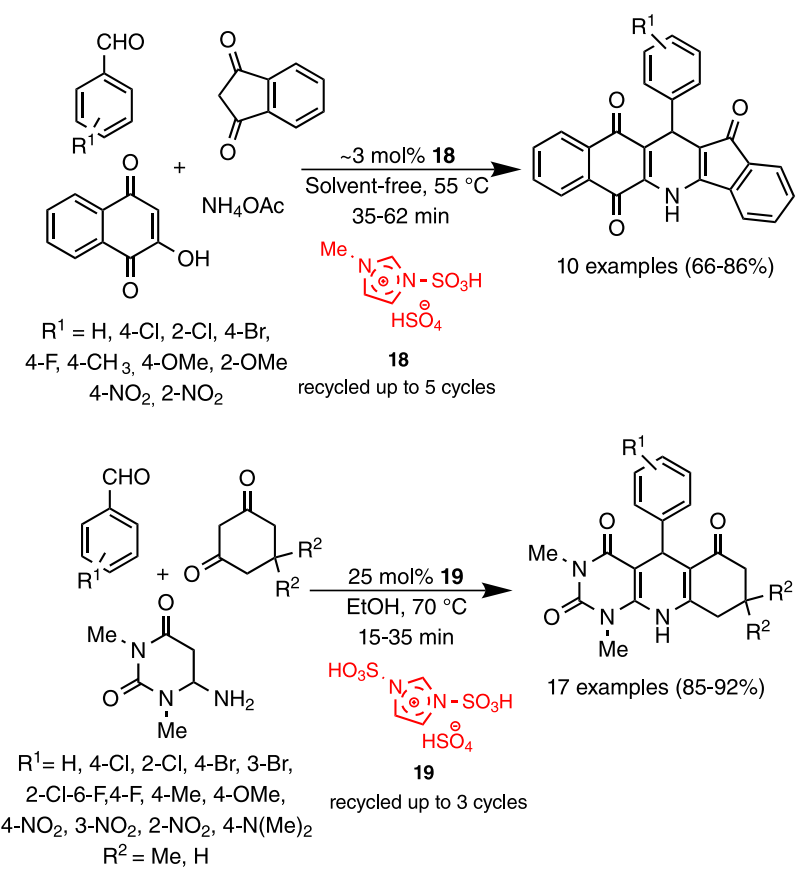

Scheme 16. Synthesis of quinoline derivatives catalyzed by $\mathbf{1 8}$ and $\mathbf{1 9}$.

Hantzsch four-component reaction can also be applied to the synthesis of hexahydroquinoline scaffolds, when a cyclic 1,3-dicarbonyl compound (i.e., dimedone or 1,3-cyclohexanedione) is used as enolizable $\mathrm{CH}_{2}$-acid component. Several studies ${ }^{63,67,79-81}$ describing this multicomponent reaction catalyzed by different BAIL catalysts (bearing one or more $-\mathrm{SO}_{3} \mathrm{H}$ groups attached to an imidazolium cation) were described elsewhere (Scheme 17). Firstly, Heravi et al. ${ }^{79}$ exploited the use of 1-methyl-3-(3-sulfobuyl)-imidazolium hydrogen sulfate 20, $\left[\left(\mathrm{SO}_{3} \mathrm{H}\right)^{4} \mathrm{C}_{4} \mathrm{C}_{1} \mathrm{Im}\right]\left[\mathrm{HSO}_{4}\right]$, in the reaction between substituted benzaldehydes, 1,3-ketoesters, dimedone and ammonium acetate, under refluxing ethanol for 20 to $120 \mathrm{~min}$, obtaining several hexahydroquinolines in good to excellent yields. Later, other research groups ${ }^{63,67,80,81}$ exploited similar greener methods for the same approach.

The reaction mechanism for the multicomponent synthesis of hexahydroquinolines is shown in Scheme 18. Firstly, dimedone is converted to its enol form and rapidly reacts with the aldehyde via an acid-catalyzed Knoevenagel condensation to furnish the adduct $\mathbf{I}$. Under the same acid catalysis conditions, 1,3-ketoester is protonated by BAIL and is readily attacked by ammonia (from ammonium acetate) to give enamine intermediate II. The reaction proceeds through a Michael addition reaction between $\mathbf{I}$ and 
II leading to the intermediate III, which is tautomerized to enaminoester IV. In the following steps, the intermediate IV affords $\mathbf{V}$ through an intramolecular condensation via nucleophilic attack of nitrogen to the activated carbonyl

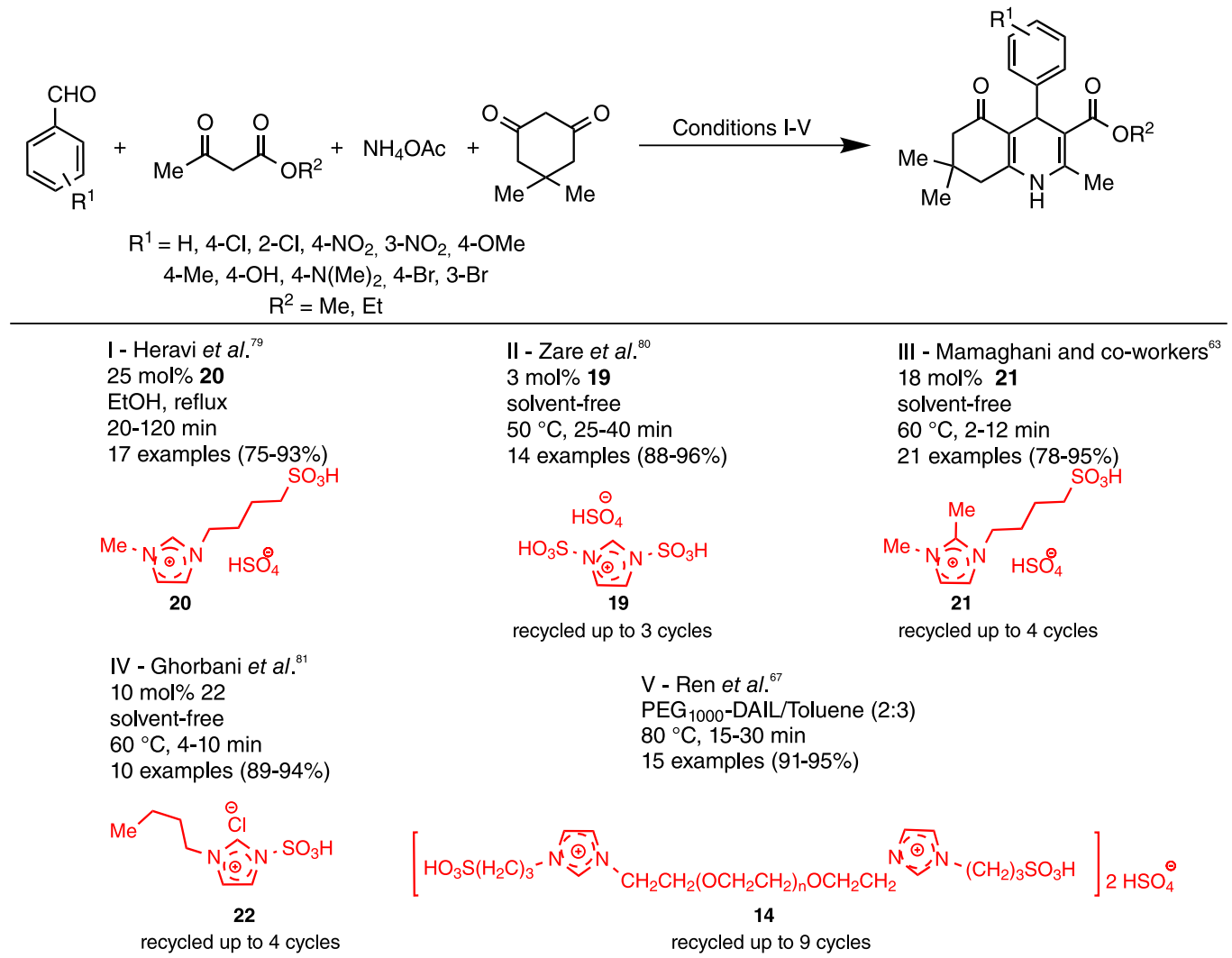

Scheme 17. Methodologies for the synthesis of hexahydroquinolines via Hantzsch four-component reaction catalyzed by $\mathrm{SO}_{3} \mathrm{H}$-functionalized imidazoliumbased catalysts 14, 19-22.
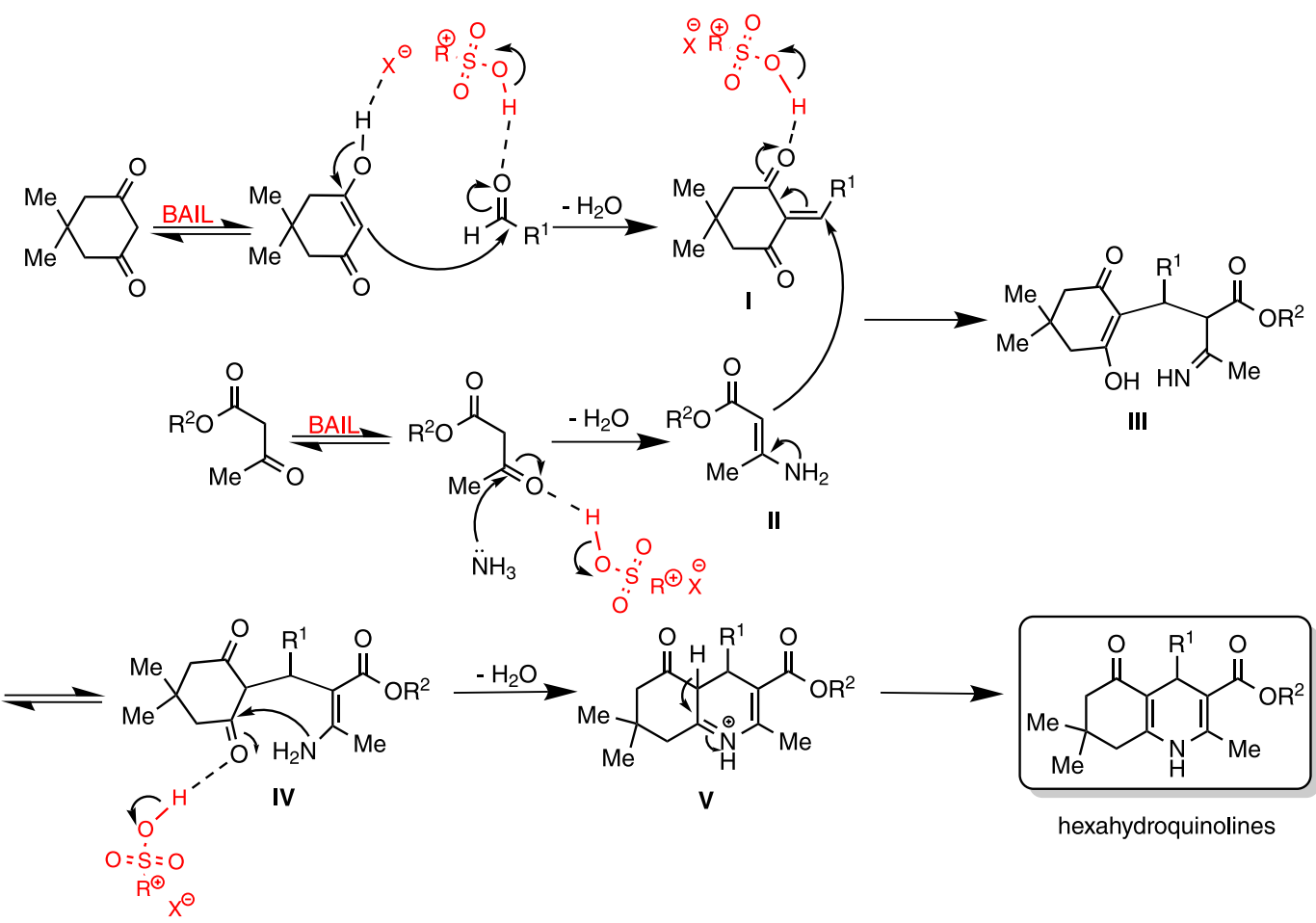

Scheme 18. Reaction mechanism for the synthesis of hexahydroquinolines catalyzed by $\mathrm{SO}_{3} \mathrm{H}$-functionalized BAILs. 
carbon, furnishing the hexahydroquinoline product after tautomerization of $\mathbf{V}{ }^{80,81}$

\subsection{Synthesis of imidazole, pyrazole and pyrrolidinone derivatives}

Imidazoles constitute an interesting class of fivemembered nitrogen heterocycles with relevant biological activities such as antihypertensive (e.g., drugs like losartan, olmesartan, eprosartan), fungicide, and herbicide. ${ }^{82,83}$ Moreover, imidazole and its derivatives are widely used as starting material for the synthesis of ionic liquids with distinct properties and applications. ${ }^{10,84}$ The synthesis of polysubstituted imidazoles catalyzed by reusable $\mathrm{SO}_{3} \mathrm{H}$-functionalized BAILs was independently investigated by two different research groups (Scheme 19). ${ }^{85,86}$ The reaction of benzil, aldehydes, primary amines and ammonium acetate, catalyzed by $\mathbf{2 3}$ or $\mathbf{1 9}$ under solventless conditions, furnished the corresponding 1,2,4,5-tetrasubstituted imidazoles in good to excellent yields. The proposed general mechanism for this multicomponent reaction catalyzed by $\mathrm{SO}_{3} \mathrm{H}$-functionalized BAILs is presented in Scheme 20.

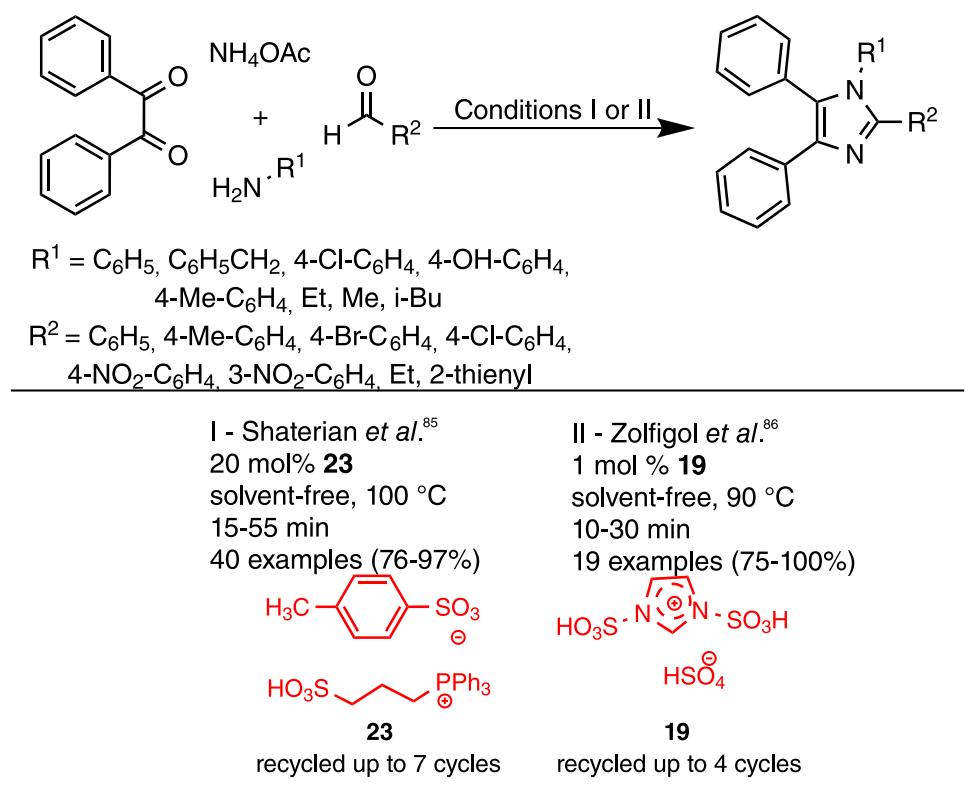

Scheme 19. Synthesis of 1,2,4,5-tetrasubstituted imidazoles via four-component reaction catalyzed by $\mathrm{SO}_{3} \mathrm{H}$-functionalized BAILs 23 and 19.

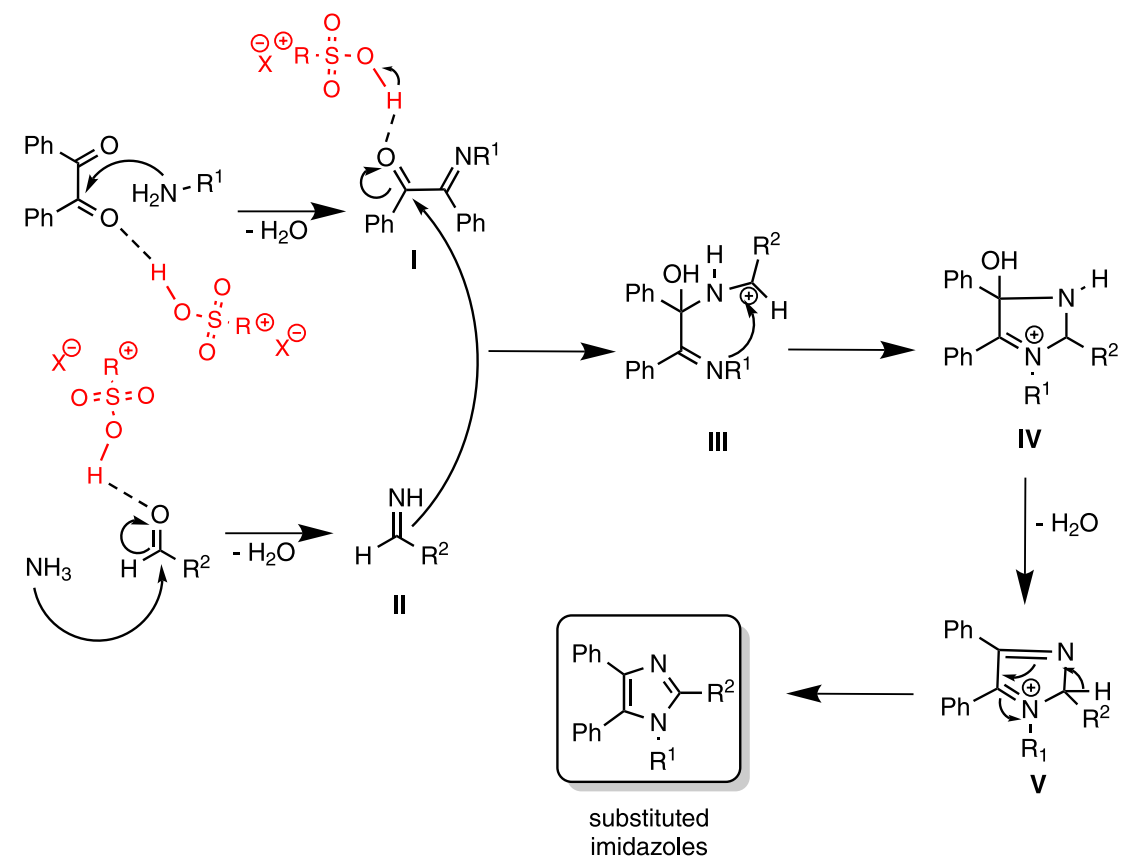

Scheme 20. Reaction mechanism for the synthesis of 1,2,4,5-tetrasubstituted imidazoles catalyzed by $\mathrm{SO}_{3} \mathrm{H}$-functionalized BAILs. 
Other contributions to the one-pot synthesis of five-membered nitrogen heterocycles catalyzed by $\mathrm{SO}_{3} \mathrm{H}$-functionalized ionic liquids involve the preparation of 2-pyrrolidinones and pyrrol-2-one derivatives. Khaligh et al ${ }^{87}$ investigated the catalytic performance of some mono- and dicationic ionic liquids bearing sulfonic acid groups as homogenous catalysts for the multicomponent reaction of anilines, aromatic aldehydes and diethyl acetylenedicarboxylate (Scheme 21). The reactions were carried out with only $1 \mathrm{~mol} \%$ of $\mathbf{2 4}$, using ethanol as solvent at room temperature for $30 \mathrm{~min}$, giving the desired products in 52-94\% yield. The catalyst 24 could be recovered and reused up to 3 reaction cycles without significant loss of activity. The authors suggested that the presence of alkyl chain linker between the imidazolium cationic heads with the sulfonic acid groups increased the catalytic activity of the BAIL. Additionally, it seems that the presence of imidazole $\mathrm{C}-2$ acidic hydrogen also enhances the catalytic efficiency by improving overall catalyst acidity. Later, the same group ${ }^{88}$ successfully applied this efficient green methodology using planetary ball milling as mechanochemical process for the synthesis of 2-pyrrolidinones. In a similar study, Yarie et al. ${ }^{89}$ described the use of tributyl(3-sulfopropyl)phosphonium hydrogen sulfate (TBSPHS) 25 as Brønsted acidic ionic liquid catalyst for the synthesis of 1,5-dihydro-2 $H$-pyrrol-2-one scaffold. Thus, the solvent-free multicomponent reaction between several aromatic aldehydes, anilines (2 equivalents) and pyruvic acid ( 2 equivalents), using $10 \mathrm{~mol} \%$ of $\mathbf{2 5}$ under solvent-free conditions at $60{ }^{\circ} \mathrm{C}$ for $15-25 \mathrm{~min}$, afforded the trisubstituted pyrrol-2-one derivatives in good yields (78-83\%) (Scheme 21).

Abshirini and Zare $^{90}$ studied the dicationic BAIL 26 as catalyst for the synthesis of bis-pyrazole derivatives using one-pot pseudo five-component reaction between arylaldehydes, phenylhydrazine and ethyl acetoacetate. The best catalyst performance was found when the reaction was carried out using $5 \mathrm{~mol} \%$ of $\mathbf{2 6}$ with ethanol as solvent at $75^{\circ} \mathrm{C}$ for $30 \mathrm{~min}$. This methodology was then successfully applied to the synthesis of several 4,4'-(arylmethylene)bis(3-methyl-1-phenyl-1 $H$-pyrazol-5-ol) derivatives in good to high yields (Scheme 22).

More recently, Rezaei et al..$^{91}$ synthesized dicationic $\mathrm{SO}_{3} \mathrm{H}$-functionalized ionic liquid immobilized on silicacoated $\mathrm{Fe}_{2} \mathrm{O}_{4}$ nanoparticles as heterogenous catalyst for the solventless three-component synthesis of bis-pyrazoles compounds. The authors evaluated different reaction parameters, and the reaction of hydrazine $\left(\mathrm{R}^{1}=\mathrm{H}\right)$ or phenylhydrazine $\left(\mathrm{R}^{1}=\mathrm{Ph}\right)$, aromatic aldehydes and ethyl acetoacetate using $10 \mathrm{mg} \mathrm{mmol}^{-1}$ of 27 under neat conditions rapidly furnished the desired products in good to excellent yields (Scheme 23). In addition, the recovery procedure of the heterogeneous catalyst $\mathbf{2 7}$ was easily carried out by using an external magnetic field, allowing it to be reused up to five times without loss of catalytic activity. A general mechanism for the BAIL-catalyzed
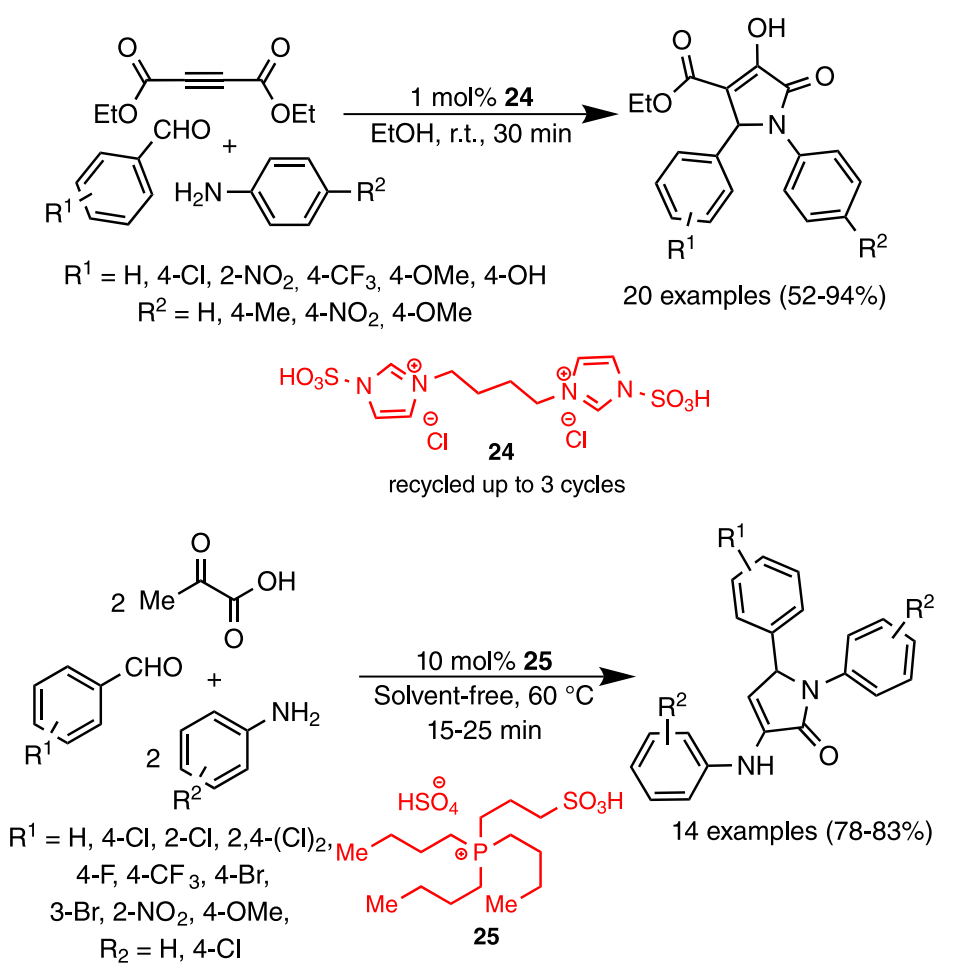

Scheme 21. BAIL-catalyzed multicomponent synthesis of 2-pyrrolidinones and pyrrol-2-ones. 


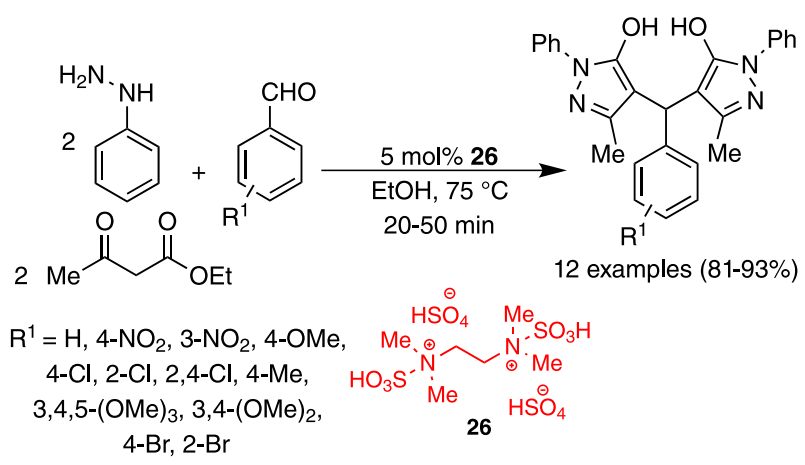

Scheme 22. A pseudo five-component reaction for the synthesis of 4,4'-(arylmethylene)-bis(3-methyl-1-phenyl- $1 H$-pyrazol-5-ol) derivatives catalyzed by $\mathrm{SO}_{3} \mathrm{H}$-difunctionalized BAIL 26.

multicomponent reaction of the synthesis of bis-pyrazoles is presented in Scheme 24..$^{91}$

\subsection{Synthesis of $4 H$-pyran derivatives and related compounds}

In the past decade, new attempts towards ecofriendly protocols for the synthesis of oxygen-containing fused heterocycles played an important role in Organic Synthesis. ${ }^{92,93}$ Organic compounds containing 2-amino4-aryl-4H-chromene-3-carbonitrile scaffold show interesting biological activities and their one-pot 3-CR (three-component reaction) synthesis usually involves a condensation reaction between arylaldehydes, 1,3-cyclic diketones (i.e., dimedone or 1,3-cyclohexanedione) and malononitrile (or cyanoacetates). ${ }^{92-94}$ In addition, distinct types of catalyst, either acidic or basic, were exploited for this reaction, including the $\mathrm{SO}_{3} \mathrm{H}$-functionalized ionic liquids. ${ }^{95}$

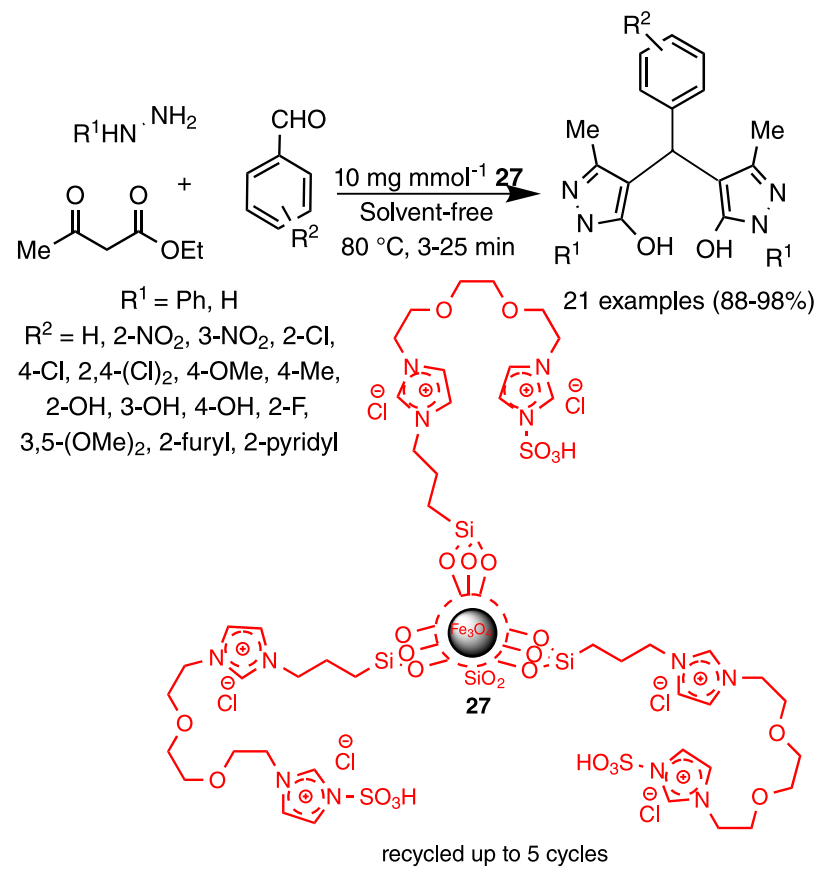

Scheme 23. Synthesis of bis-pyrazoles through MCR catalyzed by dicationic BAIL immobilized on $\mathrm{Fe}_{3} \mathrm{O}_{4} @ \mathrm{SiO}_{2} 27$.

The Brønsted acidic ionic liquid-catalyzed synthesis of 2-amino-4-aryl-4H-chromene-3-carbonitriles via MCR between dimedone, aromatic aldehydes and $\mathrm{CH}_{2}$-acid starting materials (usually malononitrile) were exhaustively studied by several research groups (Scheme 25). ${ }^{96-100}$ The $\mathrm{SO}_{3} \mathrm{H}$-functionalyzed tetraalkyl ammonium ionic liquid 27, sulfonic acid functionalized pyridinium chloride $\mathbf{2 8}$ as well as piperidinium and piperazinium-based acidic ionic liquids 29 and 30, respectively, were successfully used as catalysts for this transformation. In the most typical
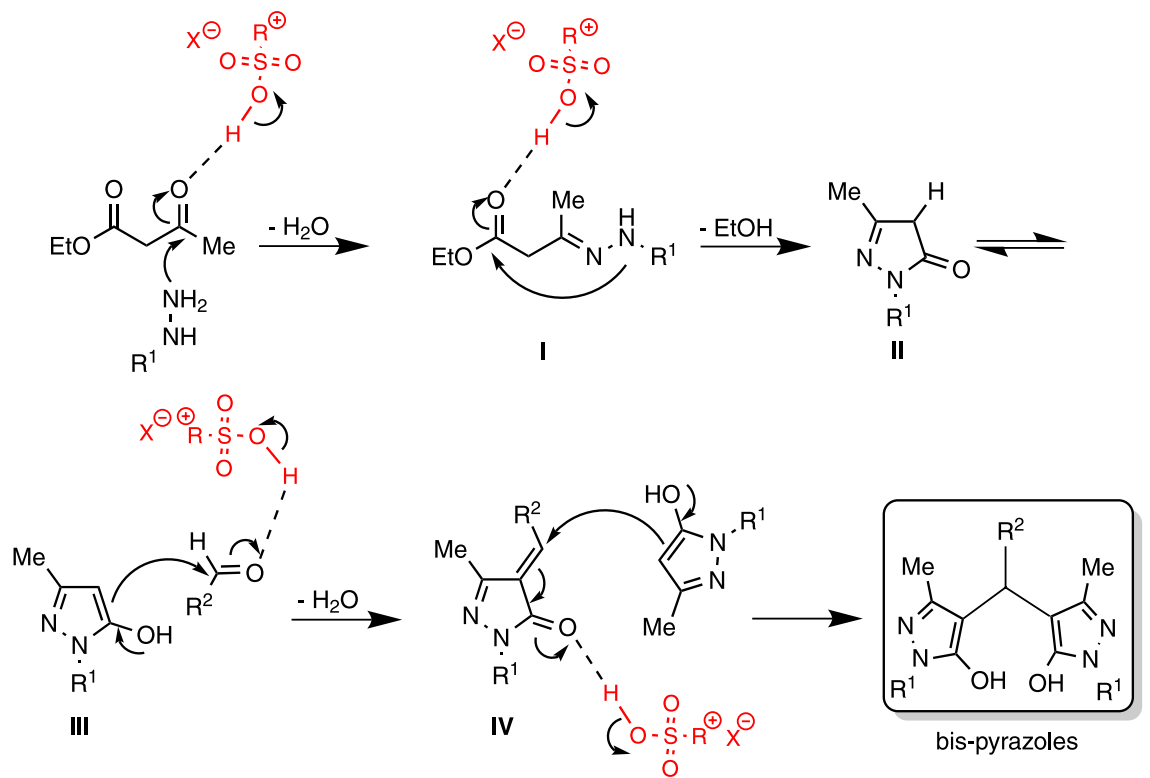

Scheme 24. Reaction mechanism for the synthesis of bis-pyrazoles catalyzed by $\mathrm{SO}_{3} \mathrm{H}$-functionalized BAILs. 
procedures, the catalyst loading varies from 3 to $10 \mathrm{~mol} \%$, usually under solventless conditions and moderate heating $\left(\mathrm{T}<100{ }^{\circ} \mathrm{C}\right)$ or room temperature. Moreover, all Brønsted acidic ionic liquid catalysts could be recycled with different catalytic efficiency and number of reaction cycles. It is noteworthy that Khaligh et al. ${ }^{99}$ employed 4,4'-trimethylene- $N, N$ '-sulfonic acid-dipiperidinium chloride 29 as homogeneous acidic catalyst for the synthesis of the title compounds using planetary ball milling process.

The general mechanism for the acidic ionic liquidcatalyzed multicomponent reaction for the synthesis of $4 H$-pyran derivatives is shown in Scheme 26. Acid-catalyzed Knoevenagel condensation between the $\mathrm{CH}_{2}$-acid (e.g., malononitrile, $\mathrm{R}^{2}=\mathrm{CN}$, or ethyl cyanoacetate, $\mathrm{R}^{2}=\mathrm{CO}_{2} \mathrm{Et}$ ) and aldehyde $\left(\mathrm{R}^{1}=\right.$ aryl) is straightforward, furnishing I which is attacked by the enol form of 1,3-dicarbonyl compound. Intermediate II undergoes cyclization to furnish III which tautomerize to the $4 H$-pyran products.

Xanthenes also constitute an important class of oxygencontaining fused heterocycles with preponderant natural occurrence, displaying a notorious interest in Medicinal Chemistry due its well-known therapeutic properties, such as anti-inflammatory, ${ }^{101}$ antibacterial, ${ }^{102}$ antiviral, ${ }^{103}$ and antitumor. ${ }^{104}$ Multicomponent synthesis of xanthene derivatives such as 9-aryl-1,8-dioxooctahydroxanthene (xanthenediones) scaffold usually involves a pseudo threecomponent transformation between arylaldehydes and two equivalents of $\mathrm{CH}_{2}$-acid component, either dimedone or 1,3-cyclehaxanedione, under acidic catalysis. Four different research groups studied this multicomponent reaction using homogenous Brønsted acidic ionic liquids. Fang et al. ${ }^{105}$ evaluated several $\mathrm{SO}_{3} \mathrm{H}$-functionalized ionic liquids with $\left[\mathrm{HSO}_{4}\right]^{-}$anion as catalysts for the synthesis of xanthenediones, and they found that $N, N, N$-trimethyl$\mathrm{N}$-propanesulfonic acid ammonium hydrogen sulfate $\mathbf{1 7}$ was the most efficient catalyst (Scheme 27). Similarly, Zolfigol et al. ${ }^{106}$ exploited $\mathrm{SO}_{3} \mathrm{H}$-difunctionalized imidazolium salts $\mathbf{3 1}$ bearing different anions such as $[\mathrm{Cl}]^{-},\left[\mathrm{PF}_{6}\right]^{-}$and $\left[\mathrm{BF}_{4}\right]^{-}$, as catalysts for the same multicomponent reaction. More recently, Ashtarian et al. ${ }^{107}$ and Sorkhi et al. ${ }^{108}$ successfully described the use of $\mathrm{SO}_{3} \mathrm{H}$-difunctionalized BAILs $\mathbf{3 2}$ and 33, respectively, as efficient and reusable catalysts for the synthesis of 9-aryl1,8-dioxooctahydroxanthene derivatives. In all cases, the desired products could be obtained in good to excellent yields under mild conditions, either in water or solventless conditions, within minutes to few hours. Generally, the recyclability of the catalyst was also studied, and they could be reused in three to seven consecutive reaction cycles, maintaining the catalytic activity.

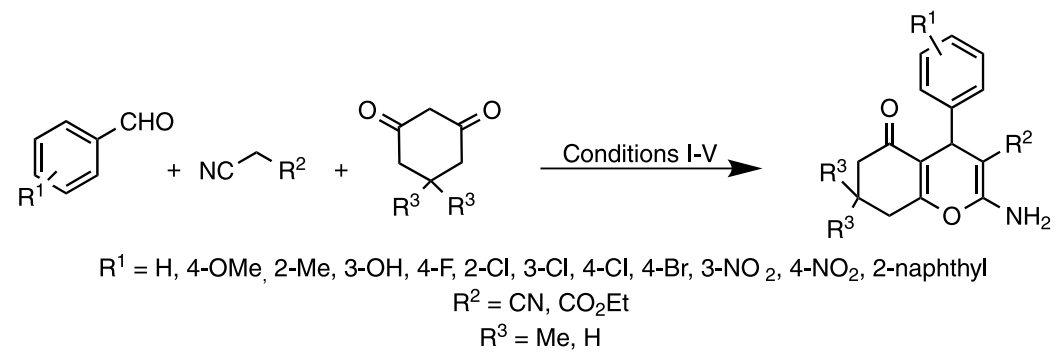

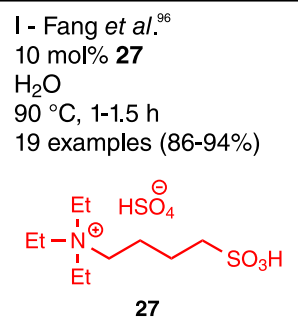

\section{II - Zolfigol et al..$^{97}$ $10 \mathrm{~mol} \% 28$}

Solvent-free $95^{\circ} \mathrm{C}, 5-20 \mathrm{~min}$ 13 examples $(86-95 \%)$

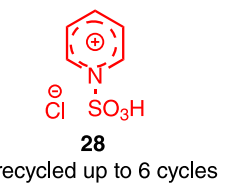

III - Sonyanaik et al ${ }^{98}$ $10 \mathrm{~mol} \% 28$ Solvent-free MW, $700 \mathrm{~W}, 2-4 \mathrm{~min}$ 14 examples $(90-94 \%)$

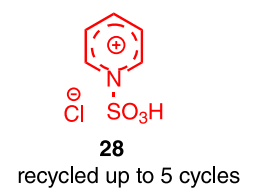

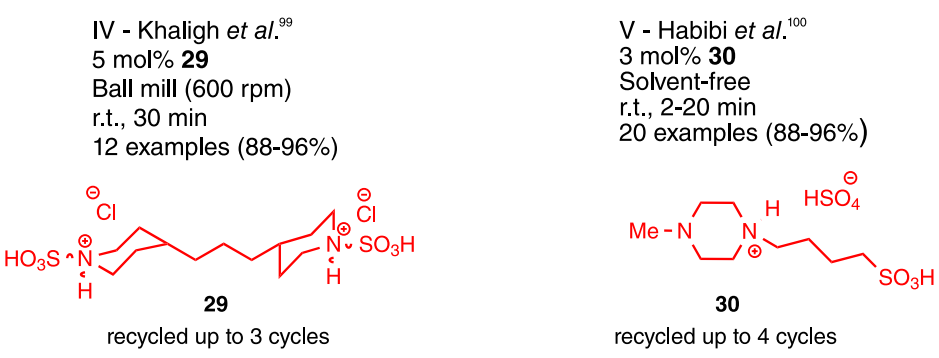

Scheme 25. Three-component synthesis of 2-amino-4-aryl-4H-chromene-3-carbonitriles catalyzed by BAIL 27-30. 


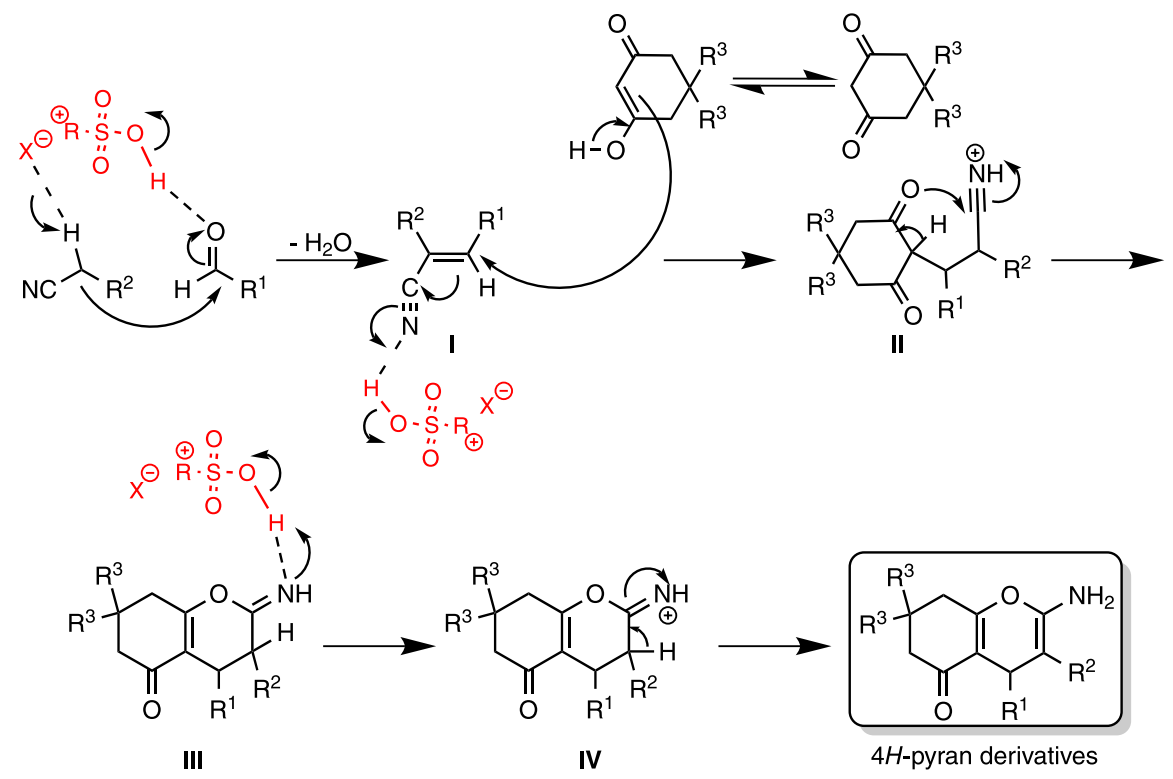

Scheme 26. Reaction mechanism for the synthesis of $4 \mathrm{H}$-pyran derivatives catalyzed by $\mathrm{SO}_{3} \mathrm{H}$-functionalized BAILs.

Other xanthene derivatives were efficiently synthesized through MCR catalyzed by $\mathrm{SO}_{3} \mathrm{H}$-functionalized BAILs. In the majority of the studies described elsewhere, the second equivalent of dimedone was replaced by other $\mathrm{CH}_{2}$-acid components, giving rise to different xanthenerelated compounds. For example, the 3-CR between $\beta$-naphthol, dimedone and aromatic aldehydes furnished a tetrahydrobenzo $[a]$ xanthene-11-one scaffold (Scheme 28). This transformation was studied by Zolfigol et al. ${ }^{106}$ as a part of the same study described in Scheme 27, in which all the acidic catalyst evaluated (i.e., 31a-31c) displayed similar catalytic efficiencies. In a similar study, Dutta et al. ${ }^{109}$ synthesized three innovative $\mathrm{SO}_{3} \mathrm{H}$-difunctionalized tetramethylguanidinium ionic liquids as reusable catalysts for the synthesis of tetrahydrobenzo[ $[a]$ xanthene11-ones. After catalytic performance tests, the ionic liquids with carboxylate anions $\mathbf{3 4 a}$ and $\mathbf{3 4 b},\left[\mathrm{CCl}_{3} \mathrm{COO}\right]^{-}$and $\left[\mathrm{CF}_{3} \mathrm{COO}\right]^{-}$, respectively, showed higher catalytic activity than those with acetate anion, suggesting that the anionic group also play a role in the catalytic efficiency. In fact, ionic liquids 34a and 34b are stronger acids due their very stable conjugated base, and stronger ionic interaction between cation/anion pairs was demonstrated by Hammett acidity function measurements and thermogravimetric analysis.

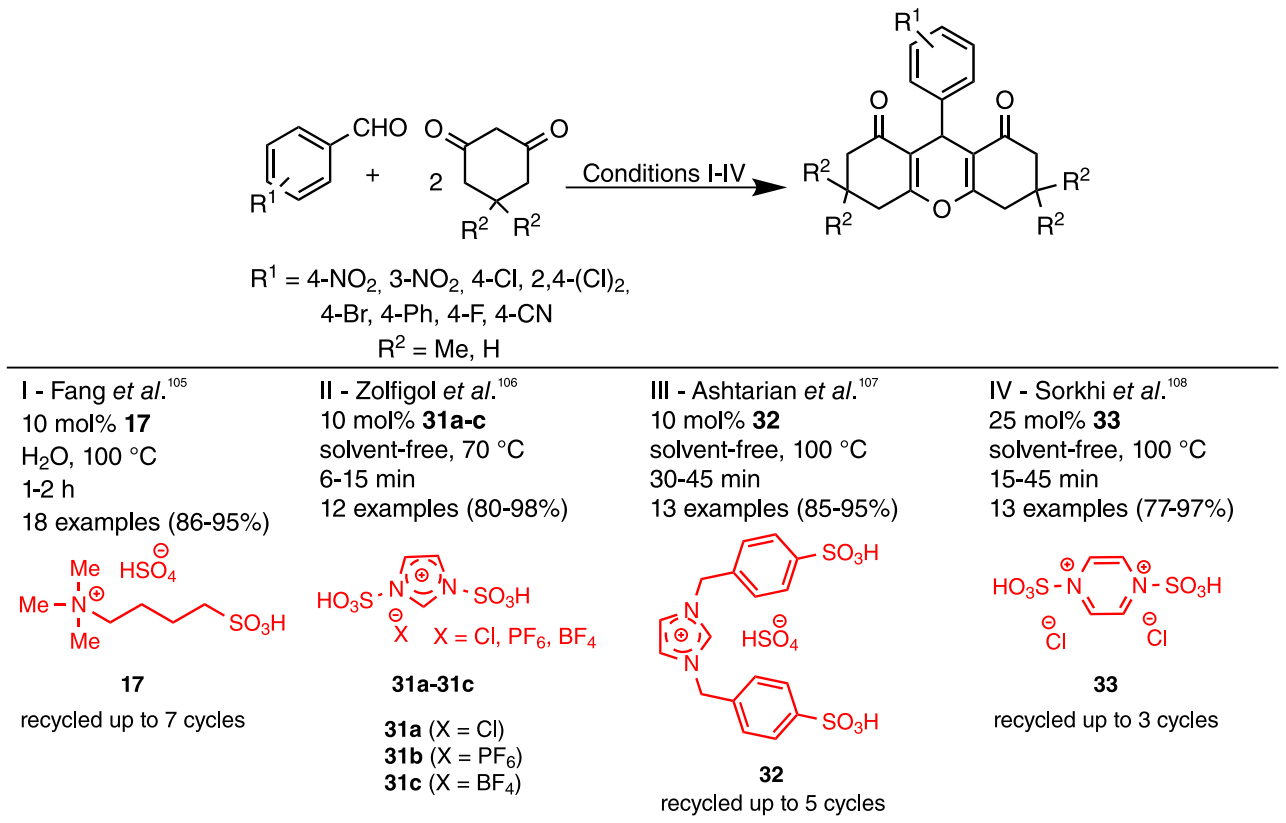

Scheme 27. Synthesis of 9-aryl-1,8-dioxooctahydroxanthene derivatives via MCR catalyzed by BAILs. 


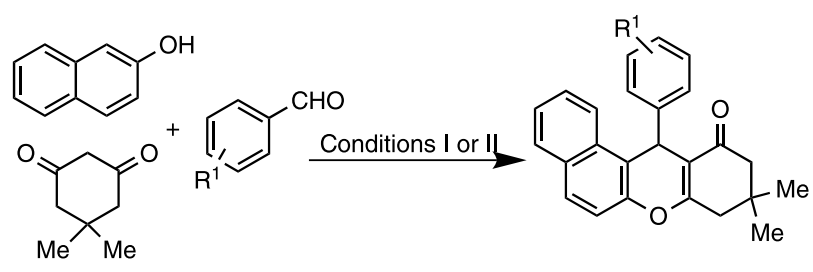

$\mathrm{R}^{1}=4-\mathrm{NO}_{2}$, 4-Cl, 4-OMe, 4-Me, 4-Br, 3-Br, 2-Br

\begin{tabular}{|c|c|}
\hline $\begin{array}{l}\text { I - Zolfigol et al. } .^{106} \\
10 \text { mol\% } 31 \mathrm{a}-31 \mathrm{c} \\
\text { Solvent-free, } 110^{\circ} \mathrm{C} \\
6-15 \text { min } \\
12 \text { examples }(83-97 \%)\end{array}$ & $\begin{array}{l}\text { II - Dutta et al. }{ }^{109} \\
5 \text { mol\% } \mathbf{3 4 a - 3 4 b} \\
\text { Solvent-free, } 75{ }^{\circ} \mathrm{C} \\
10-15 \text { min } \\
6 \text { examples }(87-95 \%)\end{array}$ \\
\hline 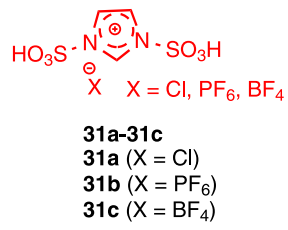 & 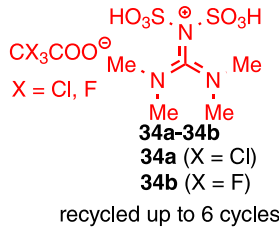 \\
\hline
\end{tabular}

Scheme 28. Synthesis of tetrahydrobenzo[a]xanthene-11-ones through MCR catalyzed by $\mathrm{SO}_{3} \mathrm{H}$-difunctionalized BAILs.

Shaterian et al. ${ }^{110}$ studied acidic ionic liquids for the three-component reaction between aromatic aldehydes, $2 H$-indene-1,3-dione, and $\beta$-naphthol or 2-hydroxynaphthalene-1,4-dione, to furnish 13-arylindeno[1,2-b]naphtha[1,2-e]pyran-12(13H)-one or 12-aryl$12 H$-indeno[1,2-b]naphtho[3,2-e]pyran-5,11,13-trione scaffolds, respectively (Scheme 29). The authors used the $\mathrm{SO}_{3} \mathrm{H}$-functionalized BAILs $\mathbf{1 1}$ and $\mathbf{3 5}$ under solvent-free conditions to obtain the title compounds in excellent yields $(90 \%)$. Later, the same group ${ }^{111}$ successfully exploited these acidic ionic liquid catalysts for a 3-CR solvent-free synthesis of xanthene derivatives.

Several research groups ${ }^{112-116}$ described methodologies

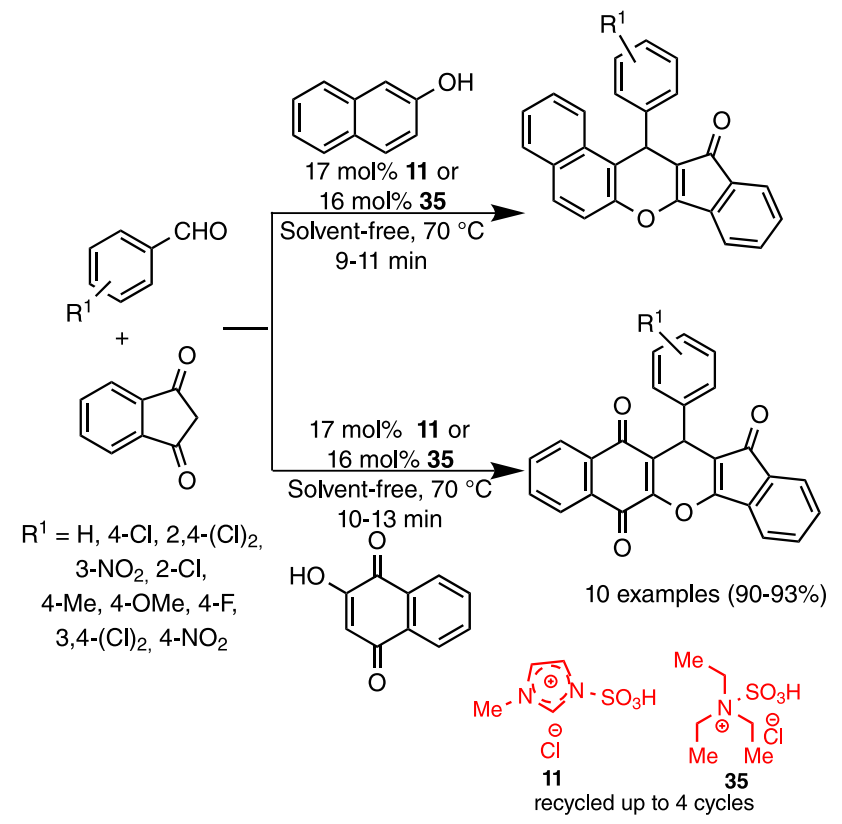

for the multicomponent synthesis of pyrano[2,3-c]pyrazoles derivatives catalyzed by different Brønsted acidic ionic liquids. Pyrano[2,3-c]pyrazole scaffold is an important class of heterocycles which can be prepared through a fourcomponent reaction between arylaldehydes, 1,3-ketoesters, hydrazine (or phenyl hydrazine), and $\mathrm{CH}_{2}$-acid reagents (usually malononitrile or dimethyl malonate).

Solvent-free approaches to pyrano[2,3-c]pyrazoles were independently studied by Ebrahimi et al. ${ }^{112}$ and Mamaghani et al., ${ }^{113,114}$ who investigated the catalytic performance of structurally related BAILs $\mathbf{2 0}$ and 21, respectively (Scheme 30). Despite the small differences on the reaction conditions used in both works, all reactions proceeded smoothly, giving rise to several pyrano[2,3-c]pyrazole derivatives in good to excellent yields. Later, Zakeri et al. ${ }^{115}$ evaluated several ordinary acidic ionic liquids based on imidazolium, pyridinium and imidazolidinium cations, as water-compatible catalysts for the synthesis of pyrano[2,3-c]pyrazoles. In the catalytic performance tests, ionic liquids bearing an alkyl acid sulfonic group were more efficient. Therefore, $\mathrm{SO}_{3} \mathrm{H}$-difunctionalized ionic liquid $\mathbf{3 6}$ was selected as the best catalyst for the synthesis of a library of pyranopyrazoles in high to excellent yields. The scope and limitations of these methodologies were also successfully exploited towards bis-pyrano[2,3-c]pyrazoles synthesis by using a bis-aldehyde component, such as isophthalaldehyde and terephthalaldehyde. ${ }^{13-115}$ Alternatively, pyrano[2,3-c]pyrazoles derivatives were prepared through a three-component condensation between arylaldehydes, malononitrile and 3-methyl-

Scheme 29. Synthesis of polycyclic $4 H$-pyrans using MCR catalyzed by different BAILs.

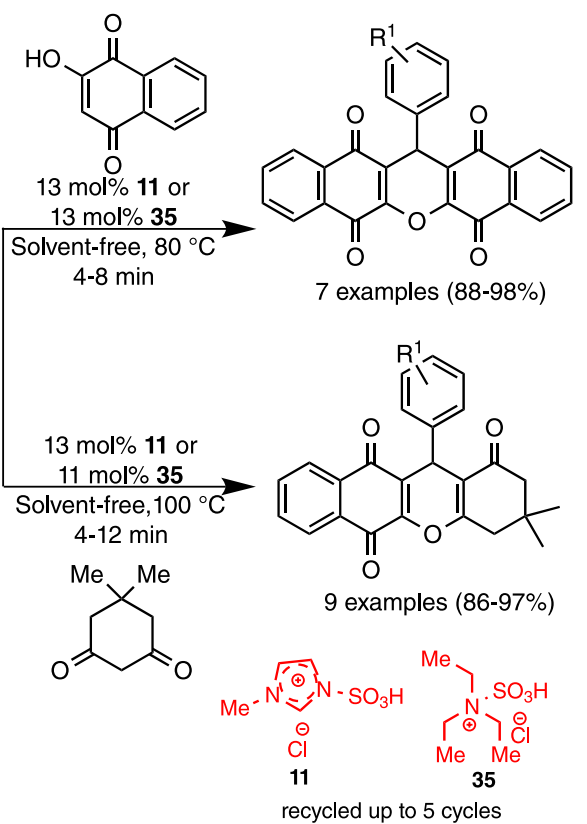




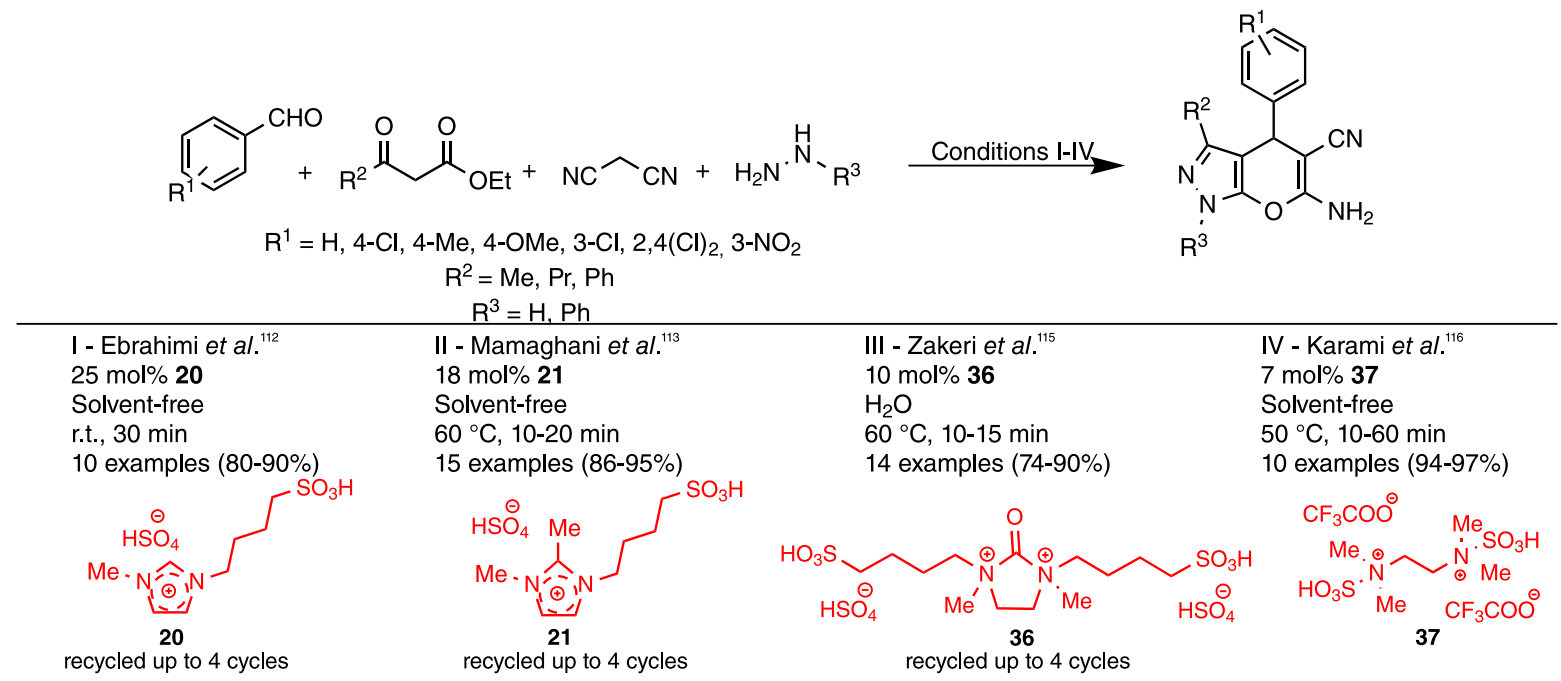

Scheme 30. Synthesis of pyrano[2,3-c]pyrazole derivatives using multicomponent reactions catalyzed by different BAILs.

1-phenyl-1 $H$-pyrazol-5(4H)-one (preformed via reaction of ethyl acetoacetate and phenylhydrazine) catalyzed by $N^{1}, N^{1}, N^{2}, N^{2}$-tetramethyl- $N^{1}, N^{2}$-bis-(sulfoethyl)1,2-diammonium bis-trifluoroacetate [TMBSED][TFA] ${ }_{2}$ 37. ${ }^{116}$

Zolfigol et al. ${ }^{117}$ prepared alkyl urea $\mathrm{SO}_{3} \mathrm{H}$-functionalized ionic liquid phases immobilized on $\mathrm{Fe}_{3} \mathrm{O}_{4} @ \mathrm{SiO}_{2}$ magnetic nanoparticles as catalyst for the one-pot synthesis of pyrano[2,3- $d$ ] pyrimidinones. The efficiency of heterogenous catalyst $\mathbf{3 8}$ was demonstrated through three-component transformations between malononitrile, arylaldehydes and 1,3-dimethylbarbituric acid, using only $0.01 \mathrm{~g} \mathrm{mmol}^{-1}$ of 38 under solventless conditions at $60{ }^{\circ} \mathrm{C}$ for 15 to $50 \mathrm{~min}$ (Scheme 31). Overall, the reactions proceeded smoothly under these optimized conditions, leading to several pyrano $[2,3-d]$ pyrimidinones in excellent yields (> 90\%). After each reaction run, the catalyst was easily recovered and it was reused up to seven consecutive cycles without significant loss in activity.

\subsection{Miscellaneous}

Phthalazine derivatives are nitrogen-based heterocycles containing a bridged hydrazine-like head which have gained attention due their potential biological properties such as anticancer, antihypertensive, antidiabetic, antiinflammatory, analgesic, antimicrobial and antidepressant, among others. ${ }^{118}$

Acid-catalyzed multicomponent approaches to synthesize organic compounds with $2 \mathrm{H}$-indazolo [2,1-b]phthalazine-1,6,11(13H)-triones scaffold were quite exploited in the literature. ${ }^{119-128}$ The standard protocols, either via three- (3-CR) or four-component reaction (4-CR) methodologies, involved the combination of non enolizable

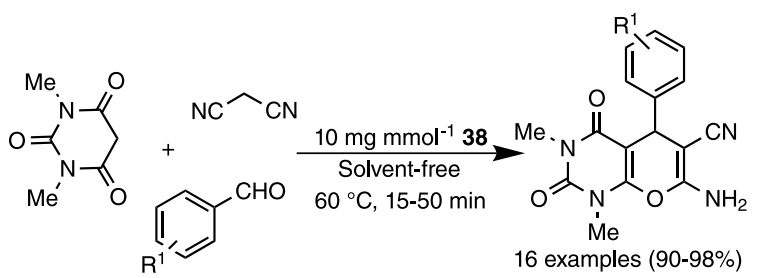

$\mathrm{R}^{1}=\mathrm{H}, 4-\mathrm{Cl}, 3-\mathrm{Cl}, 2-\mathrm{Cl}, 2,3-(\mathrm{Cl})_{2}$, 3- $\mathrm{NO}_{2}, 4-\mathrm{NO}_{2}, 4-\mathrm{Cl}-3-\mathrm{NO}_{2}$, 2-F, 4-F, 2-Br, 3-Br, 4-Br, 2-OMe, 4-OMe, 4-Me

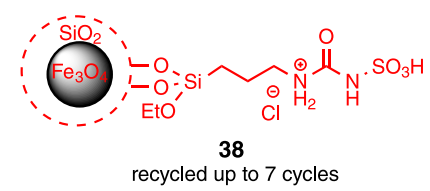

Scheme 31. Synthesis of pyrano[2,3- $d]$ pyrimidinones catalyzed by BAIL immobilized on $\mathrm{Fe}_{3} \mathrm{O}_{4} @ \mathrm{SiO}_{2}$ nanoparticles 38.

aldehydes, a $\mathrm{CH}_{2}$-acid component (usually dimedone) and phthalhydrazide, which in turn can be formed in situ through the condensation of hydrazine and phthalic anhydride (4-CR approach). So far, these relatively simple multicomponent approaches have been exhaustively used as model reactions to demonstrate the scope and limitations of several acidic ionic liquid catalysts (Scheme 32).

Three research groups ${ }^{119-121}$ described solventless 4-CR methodologies to the synthesis of $2 \mathrm{H}$-indazolo [2,1-b]phthalazine-1,6,11(13H)-triones derivatives using reusable $\mathrm{SO}_{3} \mathrm{H}$-functionalized BAILs as catalysts. Shaterian and Aghakhanizadeh ${ }^{119}$ exploited the (4-sulfobutyl) tris(4-sulfophenyl)phosphonium hydrogensulfate $\mathbf{3 9}$ and triphenyl(propyl-3-sulphonyl)phosphonium toluenesulfonate $\mathbf{4 0}$ as catalysts for the synthesis of the title compounds in high to excellent yields. Similarly, Habibi and Shamsian ${ }^{120}$ successfully applied the bifunctional acid-base ionic liquid 1,4-dimethyl(4-sulfobutyl)piperazinium hydrogen sulfate $\mathbf{4 1}$ as catalyst for rapid and efficient synthesis of $2 H$-indazolo[2,1- $b]$ phthalazine-1,6,11(13H)-triones derivatives. In the protocol developed by Pouramiri 


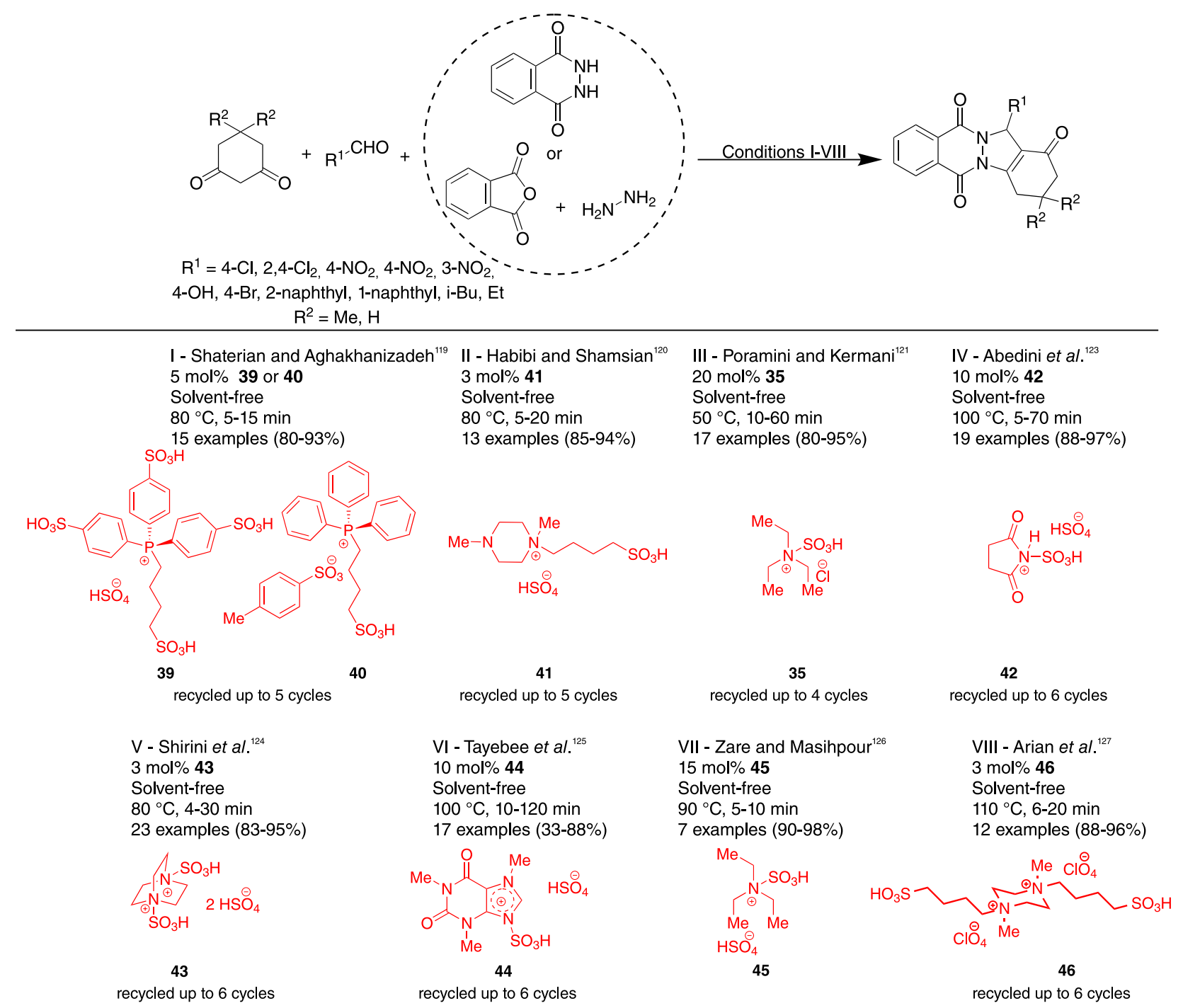

Scheme 32. Synthesis of $2 H$-indazolo[2,1-b]phthalazine-1,6,11(13H)-triones through 3-CR or 4-CR approaches catalyzed by BAILs.

and Kermani, ${ }^{121}$ the ionic liquid $\mathbf{3 5}$ was selected as the best catalyst to construct a library of $2 \mathrm{H}$-indazolo [2,1-b]phthalazine-1,6,11(13H)-triones derivatives. Later, the same group ${ }^{122}$ reported the synthesis of pyrazolo [1,2-b]phthalazine-2-carboxylate derivatives catalyzed by $\mathrm{SO}_{3} \mathrm{H}$-difunctionalized imdazolium-based ionic liquids, using alkyl acetoacetates as $\mathrm{CH}_{2}$-acid component.

Likewise, Abedini et al. ${ }^{123}$ prepared the ionic liquid succinimidinium $N$-sulfonic acid hydrogen sulfate $\mathbf{4 2}$ as new catalyst for a reaction between 1,3-cyclic diketones, phthalhydrazide and arylaldehydes (Scheme 32). In the same year, Shirini et al..$^{124}$ employed the dicationic $\mathrm{SO}_{3} \mathrm{H}$-functionalized Brønsted acidic ionic liquid $\mathbf{4 3}$ in the synthesis of several $2 H$-indazolo[2,1-b]phthalazine1,6,11(13H)-triones derivatives in excellent yields. Using established condition VI, the somewhat innovative caffeinederived ionic liquid $\mathbf{4 4}$ could be used as efficient and reusable catalyst for this reaction. ${ }^{125}$ Finally, catalysts $\mathbf{4 5}^{126}$ and $\mathbf{4 6}^{127}$ were also evaluated for the 3-CR synthesis of $2 H$-indazolo[2,1-b]phthalazine-1,6,11(13H)-triones.

A new strategy based on the use of immobilized Brønsted acidic ionic liquids for the three-component synthesis of $2 H$-indazolo[2,1- $b]$ phthalazine-1,6,11(13H)-triones was reported by Safaei et al. ${ }^{128}$ Initially, the authors demonstrated the higher catalytic efficiency of nano$\mathrm{SiO}_{2}$ supported acidic ionic liquid 47, in comparison to non-immobilized imidazolium-based ionic liquids. Thus, the heterogeneous BAIL@nano- $\mathrm{SiO}_{2}$ catalyst 47 was used for the multicomponent reaction of dimedone, aromatic aldehydes and phthalhydrazide, furnishing several $2 H$-indazolo[2,1- $b]$ phthalazine-1,6,11(13H)-triones in good to excellent yields. After each reaction run, the catalyst could be recovered and reused up to 8 cycles without significant loss in activity (Scheme 33).

Some research groups ${ }^{129-131}$ described solvent-free protocols for the one-pot synthesis of 1-amidoalkyl- 


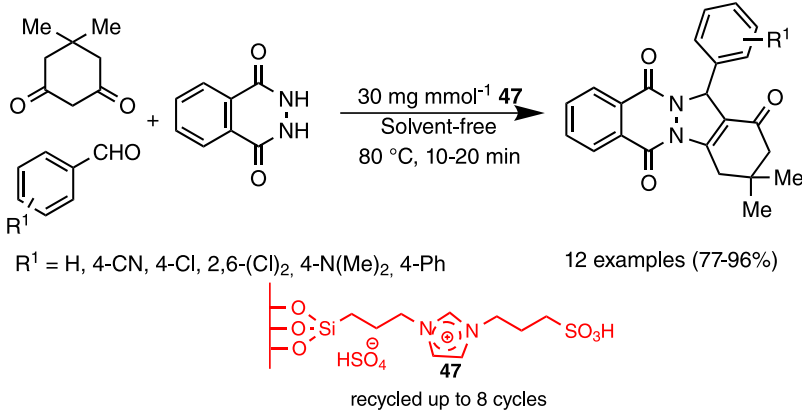

Scheme 33. Synthesis of $2 H$-indazolo[2,1- $b]$ phthalazine-1,6,11(13H)triones through 3-CR catalyzed by immobilized BAIL 47.

2-naphthols via MCR catalyzed by $\mathrm{SO}_{3} \mathrm{H}$-functionalized BAILs bearing hydrogen sulfate as anion. In general, the reactions between aromatic or aliphatic aldehydes, amides (or carbamates) and $\beta$-naphthol were carried out using 3 or $5 \mathrm{~mol} \%$ of the BAIL catalyst at moderate heating for few minutes, furnishing the desired products in excellent yields (Scheme 34).

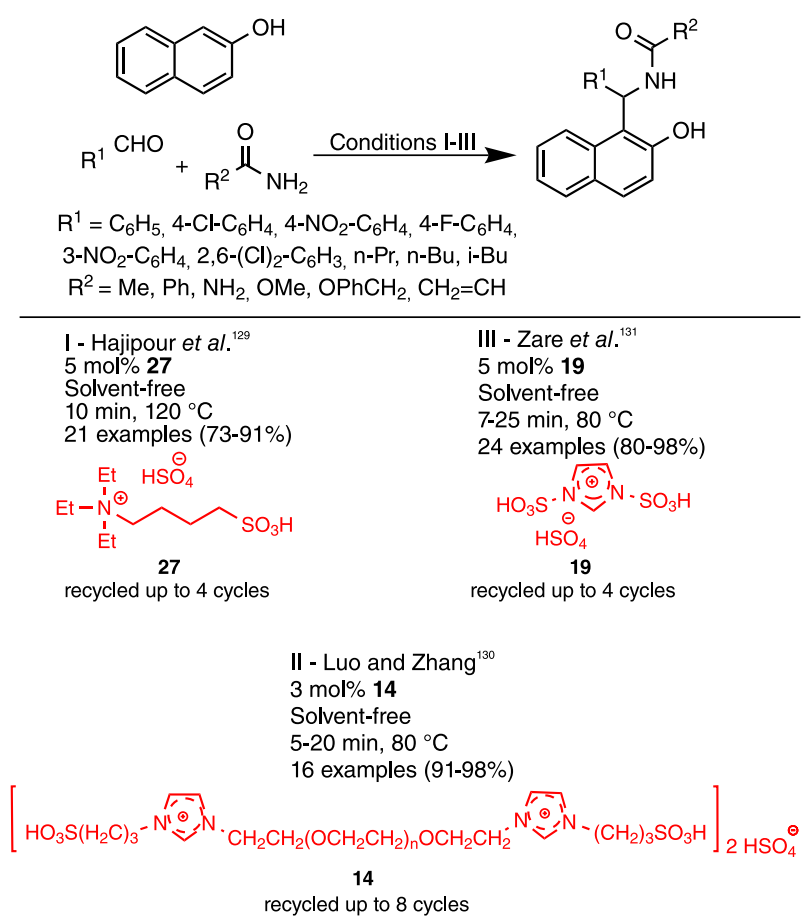

Scheme 34. Synthesis of 1-amidoalkyl-2-napthols through MCR catalyzed by $\mathrm{SO}_{3} \mathrm{H}$-functionalized BAILs.

Using a heterogeneous catalysis approach, Kotadia and $\mathrm{Soni}^{132}$ exploited an $\mathrm{SO}_{3} \mathrm{H}$-functionalized ionic liquid based on benzimidazolium salt immobilized on silica-gel support towards the multicomponent synthesis of 1-amidoalkyl naphthols. Thus, the reaction between $\beta$-naphthol, arylaldehydes and amides/urea, catalyzed by $4 \mathrm{mg} \mathrm{mmol}{ }^{-1}$ of $\mathbf{4 8}$ under solvent-free conditions, rapidly furnished several 1-amidoaldkyl naphthols in good to excellent yields (Scheme 35). According to the authors, the method represents a fast, simple and cost-effective route to the title compounds, as the catalyst could be easily separated from the reaction media, recovered and reused up to five reaction runs.

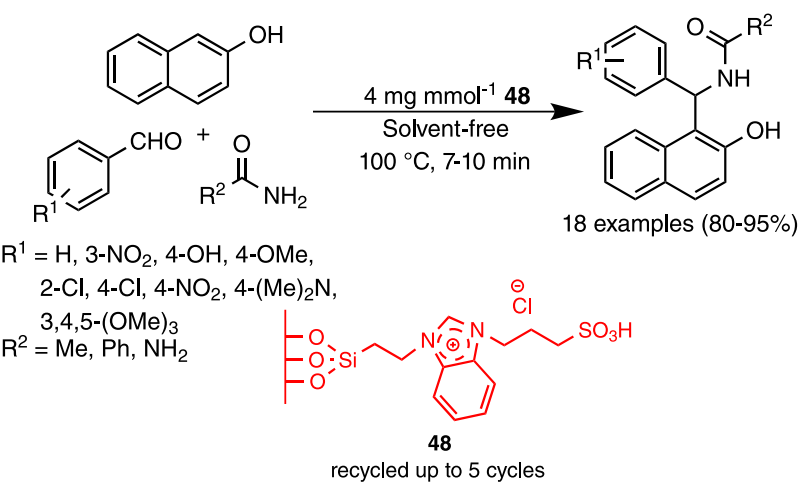

Scheme 35. Synthesis of 1-amidoalkyl naphthols through MCR catalyzed by BAIL immobilized on silica-gel support $\mathbf{4 8}$.

The preparation of $\beta$-acetamido ketone scaffolds can also be catalyzed by $\mathrm{SO}_{3} \mathrm{H}$-functionalized acidic ionic liquids. Davoodnia et al. ${ }^{133,134}$ firstly exploited the use of acidic ionic liquid $\mathbf{2 0}$ as solvent and catalyst for the reaction of aromatic aldehydes, substituted acetophenones, acyl chloride (or trimethylsilyl chloride) and acetonitrile (Scheme 36). Wang et al. ${ }^{135}$ evaluated the role of $\mathrm{SO}_{3} \mathrm{H}-$ difunctionalized ionic liquids based on piperazinium cation for the synthesis of $\beta$-acetamido ketones. Hammet function and minimum-energy geometry calculations for all $\mathrm{SO}_{3} \mathrm{H}$-difunctionalized ionic liquids bearing different anions revealed that BAILs with shorter $\mathrm{O}-\mathrm{H}$ bond lengths are stronger acids and, therefore, better catalysts. Thus, the catalyst 49 was chosen as the best catalyst for this transformation, leading to several $\beta$-acetamido ketones in high yields. Similarly, Zare et al. ${ }^{136}$ exploited the use of ammonium salt $\mathbf{3 5}$ as catalyst for the multicomponent synthesis of $\beta$-acetamido ketones and xanthenes derivatives.

The alkyl sulfonic acid-functionalized cationic poly(4-vinylpyridine) polymer $\mathbf{5 0}$ was synthesized and studied as catalyst for the four-component synthesis of $\beta$-acetamido ketones (Scheme 37). ${ }^{137}$ Thus, the heterogeneous catalyst $\mathbf{5 0}$ was evaluated in the reactions between arylaldehydes, acetophenone, acetyl chloride and acetonitrile, leading to several $\beta$-acetamido ketones in excellent yields. It is worthy to mention that reactions did not afford the desired products when enolizable propionaldehyde was used as carbonyl component.

The investigation of $\mathrm{SO}_{3} \mathrm{H}$-funtionalized ionic liquids as catalysts in MCRs for the synthesis of several different nitrogen-based heterocyclic scaffolds was reported elsewhere. ${ }^{138-144}$ For example, the synthesis of isoxazole 


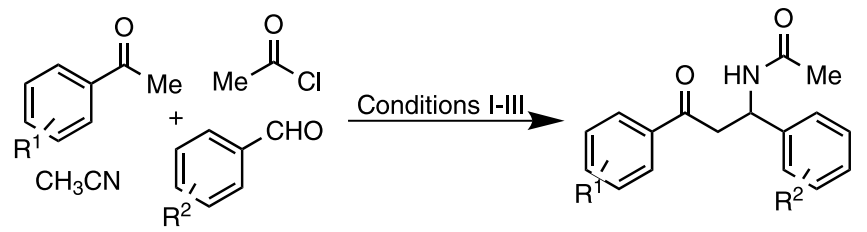

$\mathrm{R}^{1}=\mathrm{H}, 4-\mathrm{Cl}, 4-\mathrm{NO}_{2}, 4-\mathrm{CH}_{3}, 4-\mathrm{NO}_{2}$

$\mathrm{R}^{2}=\mathrm{H}, 4-\mathrm{Cl}, 3-\mathrm{Cl}, 4-\mathrm{NO}_{2}, 3-\mathrm{NO}_{2}, 4-\mathrm{Me}, 3-\mathrm{Me}$
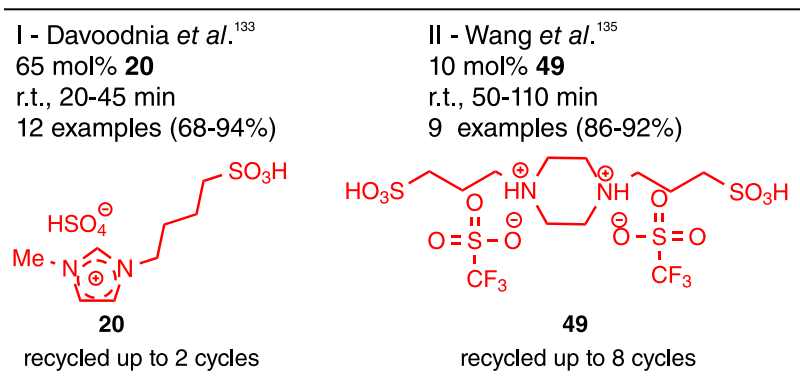

III - Zare et al. ${ }^{136}$

$15 \mathrm{~mol} \% 35$

r.t., $50-130 \mathrm{~min}$

15 examples (84-95\%)

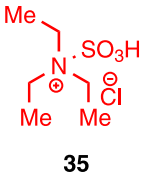

35

Scheme 36. Synthesis of $\beta$-acetamido ketones through MCR catalyzed by $\mathrm{SO}_{3} \mathrm{H}$-functionalized BAILs.

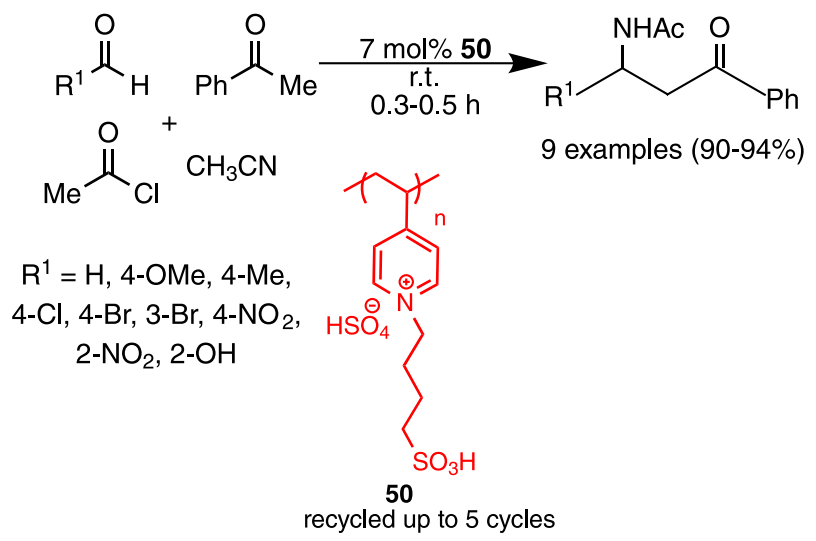

Scheme 37. Synthesis of $\beta$-acetamido ketones through four-component synthesis catalyzed by cationic alkyl $\mathrm{SO}_{3} \mathrm{H}$-functionalized poly(4vinylpyridine) polymer $\mathbf{5 0}$.

five-membered ring heterocycles via one-pot 3-CR between arylaldehydes, ethyl acetoacetate and hydroxylamine hydrochloride can be catalyzed by dicationic ionic liquid $N, N, N^{\prime}, N^{\prime}$-tetramethyl- $N, N^{\prime}$-bis(sulfo)ethane1,2-diaminium mesylate $\mathbf{5 1}{ }^{138}$ The reactions were carried out using $10 \mathrm{~mol} \%$ of $\mathbf{5 1}$ at $70{ }^{\circ} \mathrm{C}$ under neat conditions, affording several 3-methyl-4-arylmethylene-isoxazole$5(4 H)$-ones in good to excellent yields (Scheme 38).

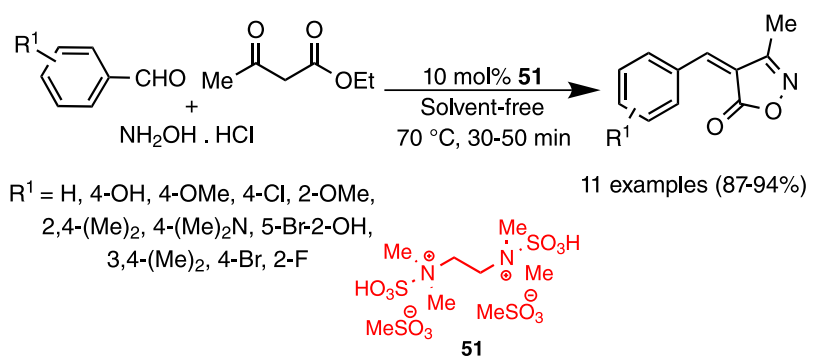

Scheme 38. Synthesis of 3-methyl-4-arylmethylene-isoxazole-5(4H)-ones through MCR catalyzed by BAIL $\mathbf{5 1}$.
Davoodnia et al. ${ }^{139}$ exploited the use of $\mathbf{2 0}$ as catalyst for the solvent-free 4-CR synthesis of substituted 2 -aminopyridines and $2(1 H)$-pyridinones. A mixture of 3,4-dimetoxyacetophenone, aromatic aldehydes, ammonium acetate and malononitrile (or ethyl cyanoacetate) was heated at $150{ }^{\circ} \mathrm{C}$ in the presence of $10 \mathrm{~mol} \%$ of $\mathbf{2 0}$ for few minutes, affording several 2-aminopyridines or 2(1H)-pyridinones in excellent yields, when malononitrile or ethyl cyanoacetate were used, respectively (Scheme 39). Similarly, Banothu et al. ${ }^{140}$ reported an efficient methodology for the solventless synthesis of several coumarin-conjugated 2-amino3-cyanopyridines catalyzed by a $\mathrm{SO}_{3} \mathrm{H}$-functionalized ionic liquid based on phosphonium salt 39 .

More recently, Zaharani et al. ${ }^{141}$ studied the low-viscous acidic ionic liquid catalyst 29 in the multicomponent synthesis of triazolopyrimidine derivatives. One advantage of using dual role ionic liquids with low viscosity comes from the possibility of performing reactions at room temperature, improving energy efficiency. In the presence of $1 \mathrm{~mL}$ of $\mathbf{2 9}$ under mild conditions, the reactions between 3-amino-1,2,4-triazole, substituted benzaldehydes and ethyl cyanoacetate proceeded smoothly, leading to dihydro$[1,2,4]$ triazolo[1,5- $a]$ pyrimidines in good to excellent yields (Scheme 40). Likewise, Basirat et al. ${ }^{142}$ described an efficient methodology for the synthesis of several nitrogencontaining fused heterocycles (e.g., triazoloquinazolinones and benzoimidazopyrimidines derivatives) via solventless three-component synthesis catalyzed by 1,3-disulfonic acid imidazolium trifluoroacetate ([Dsim] $\left[\mathrm{CF}_{3} \mathrm{CO}_{2}\right]$ ).

Mohammadi and Abbasi ${ }^{143}$ investigated the use of sulfonic acid pyridinium hydrogen sulfate $\mathbf{5 2}$ as reusable catalyst for the one-pot three-component synthesis of highly functionalized pyridines. Catalytic performance 


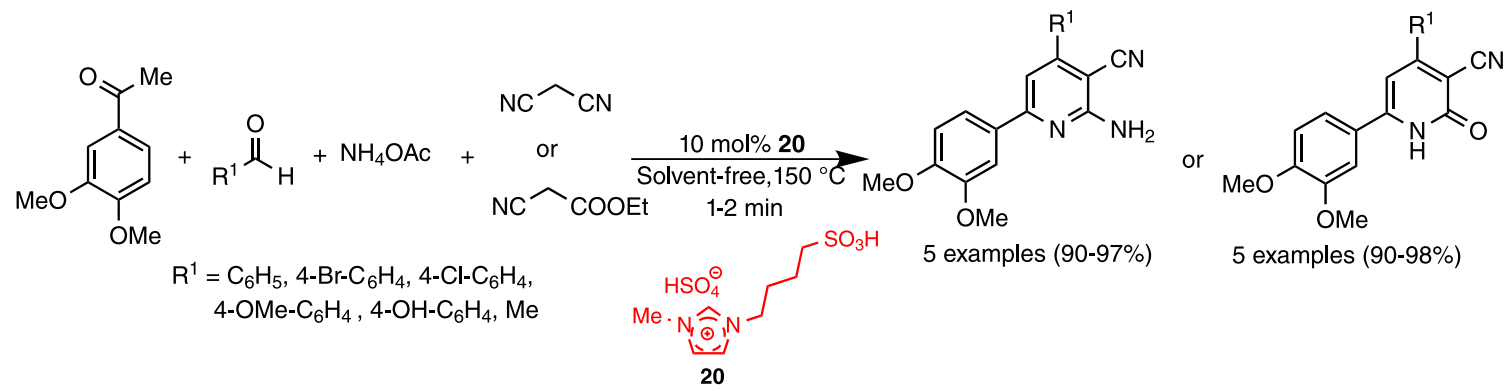

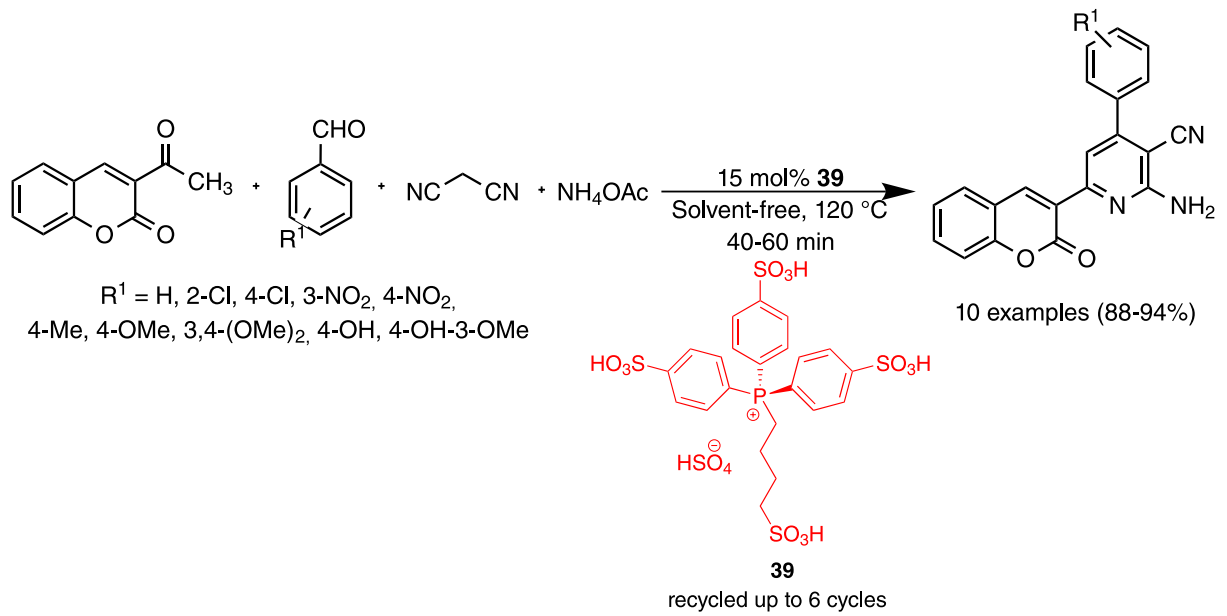

Scheme 39. Synthesis of highly substituted 2-amino-3-cyanopyridines using acidic ionic liquid catalysts 20 and 39.

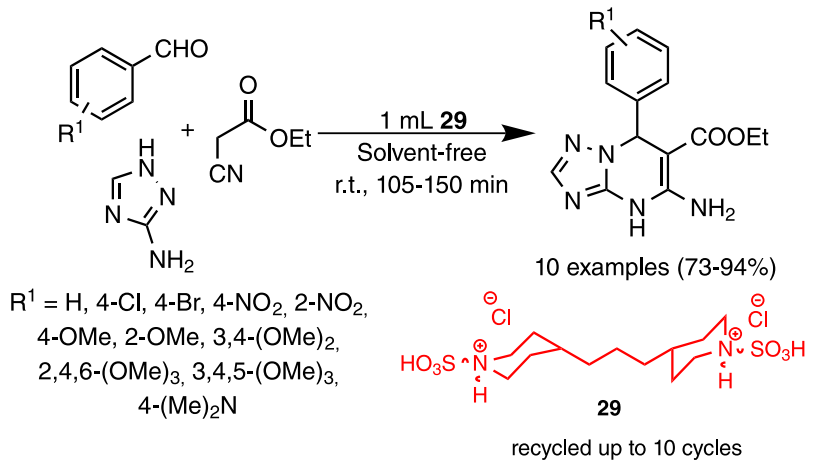

Scheme 40. Synthesis of dihydro-[1,2,4]triazolo[1,5-a]pyrimidines via MCR catalyzed by BAIL 29.

tests showed that $15 \mathrm{~mol} \%$ of $\mathbf{5 2}$ efficiently catalyzed the reaction between $p$-tolualdehyde, aniline, and ethyl acetoacetate, carried out under solventless conditions at
$100{ }^{\circ} \mathrm{C}$ within $40 \mathrm{~min}$. Then, the protocol was applied for the synthesis of highly functionalized pyridines, varying the structure of the arylaldehyde and/or the arylamine components (Scheme 41). After each reaction run, the catalyst could be recovered and reused up to five times without noticeably loss in activity.

In the methodology described by Vahdat and Akbari, ${ }^{144}$ an $\mathrm{SO}_{3} \mathrm{H}$-multifunctionalized ionic liquid based on phosphonium salt 39 was studied as catalyst for the multicomponent synthesis of acridine-1,8-diones. Using a model reaction between dimedone, 4-chlorobenzaldehyde and $p$-toluidine, the authors defined a catalyst loading of $1 \mathrm{~mol} \%$ and water as the best solvent. Thus, a library of twenty-five acridine-1,8-dione derivatives could be prepared using this simple and highly efficient method (Scheme 42). Similar studies ${ }^{109,145}$ on multicomponent

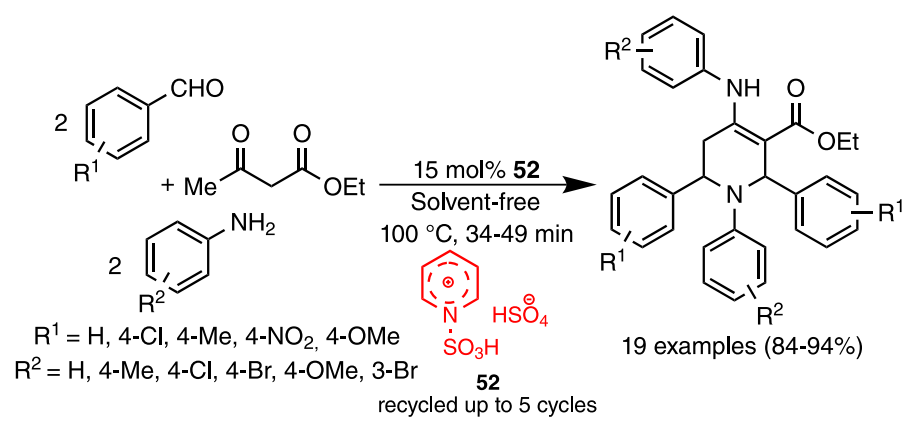

Scheme 41. Synthesis of highly functionalized pyridines through one-pot three-component synthesis catalyzed by BAIL $\mathbf{5 2}$. 
synthesis of acridine-based derivatives using of $\mathrm{SO}_{3} \mathrm{H}$ functionalized BAILs as catalysts were also published elsewhere.

Multicomponent synthesis of structural diverse spiro heterocycles catalyzed by $\mathrm{SO}_{3} \mathrm{H}$-functionalized BAILs were reported by Kumar et al. ${ }^{146}$ The authors developed an efficient reaction system applied to the four-component reaction between isatine, substituted 2 -aminobenzothiazoles and two different $\mathrm{CH}_{2}$-acid components (i.e., dimedone and 1,3-dimethylbarbituric acid). The reactions were carried out with a mixture of catalyst 20/water (1:2), respectively, at $80{ }^{\circ} \mathrm{C}$ for $15 \mathrm{~min}$, furnishing the desired spiro products in high yields (Scheme 43, selected example). Also, Seyyedhamzeh et al. ${ }^{147}$ synthesized the guanidinium-based sulfonic acid ionic liquid $\mathbf{5 3}$ as acidic catalyst for the multicomponent synthesis of several nitrogen-containing heterocycles, including the synthesis of spiro triazolo[1,2- $a$ ] indazole-tetralone derivatives in good yields (Scheme 43, selected example).

\section{Conclusions}

In this review contribution we discussed and compared several methodologies exploiting the catalytic activity of homogeneous and heterogeneous sulfonic acidfunctionalized task-specific ionic liquids in multicomponent reactions for the synthesis of nitrogen- and oxygen-based heterocycles, among others. In general, structurally diverse $\mathrm{SO}_{3} \mathrm{H}$-functionalized Brønsted acidic ionic liquids have been acknowledged as powerful catalysts for MCRs,

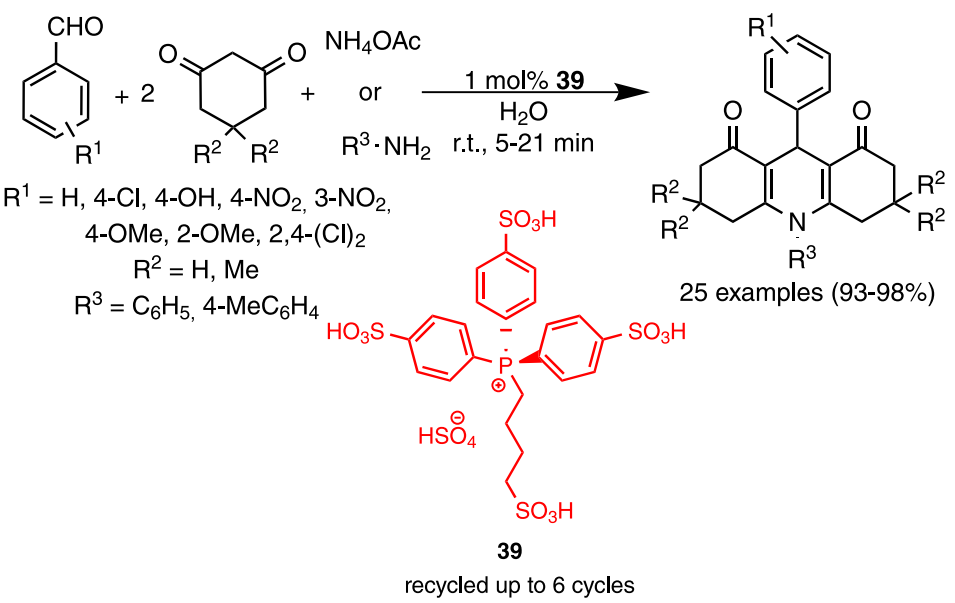

Scheme 42. Synthesis of 1,8-dioxo-decahydroacridines through MCR catalyzed by BAIL 39.

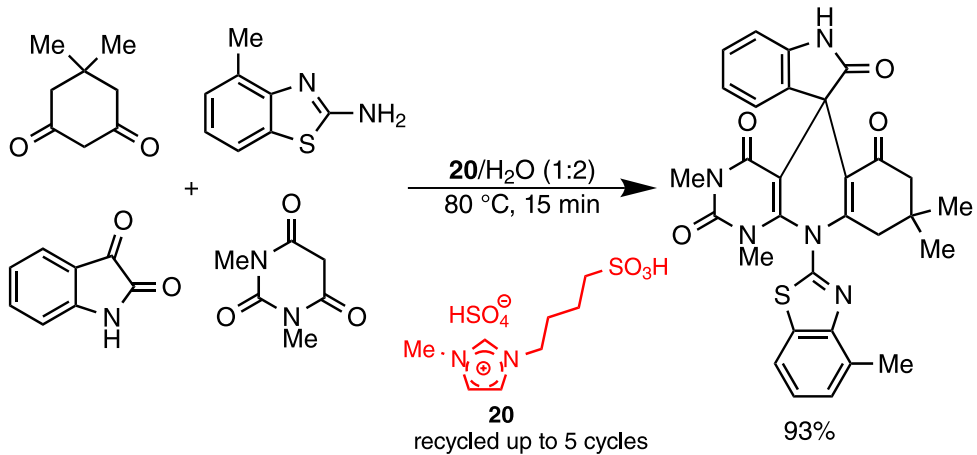<smiles>CC1(C)[CH+]C(=O)CC(=O)C1</smiles>

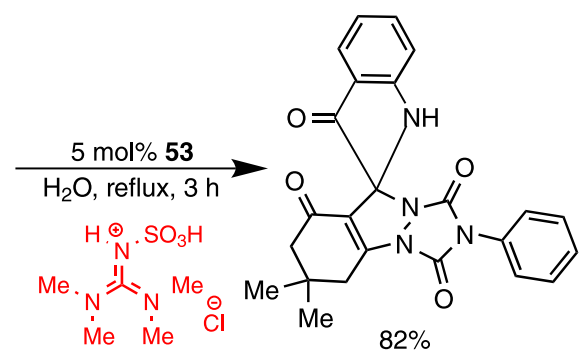


usually requiring low catalyst loading and mild conditions. Indeed, most of these catalysts showed remarkable catalytic efficiency, great compatibility with starting materials bearing different functional groups, simplified product separation methods as well as easy recovery procedures, boosting the well-known green credentials of multicomponent reactions. Although several studies claimed some advantages towards sustainable organic synthesis, the lack of implementation of green metrics for the whole process remains as obstacle for the quantification of environmental progress. It is noteworthy that the use of $\mathrm{SO}_{3} \mathrm{H}$-functionalized ionic liquids as catalysts for acidcatalyzed isocyanide-based multicomponent reactions is still under exploited. In addition, there is space for the study of BAILs as catalysts in sequential multicomponent reaction approaches, shortening the synthesis of more structural-complex scaffolds with potential biological activities. Finally, improvements and investigantions on the heterogenization of ionic liquids for acidic catalysis could also contribute to the development of even more efficient and sustainable synthetic processes.

\section{Acknowledgments}

The authors gratefully acknowledge FAPESP grants (2018/15038-7; 2018/21131-0). N. S. Anjos also acknowledges a master fellowship from CAPES (PPGQ-CTS, UNIFESP).

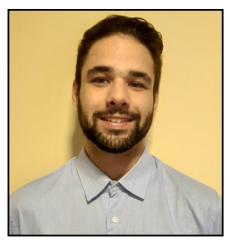

Nicolas S. Anjos obtained his BSC in Pharmacy (2019) from Federal University of São Paulo, Brazil. He joined Prof Luiz Longo research group in 2016 as an undergraduate student and, more recently as a graduate student in Chemistry, Sustainable Science and Technology. His research interests involve the study of Bronsted acidic ionic liquids as catalysts in multicomponent reactions applied to the synthesis of nitrogen-based heterocycles with potential antitumor activity.

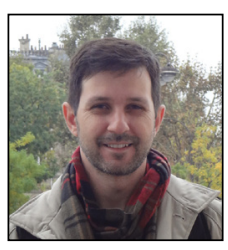

Luiz S. Longo Jr. graduated in Pharmacy and received his PhD degree in Organic Chemistry from the University of São Paulo, Brazil. In 2013, he joined the Ionic Liquid Group at The University of Nottingham/UK as postdoctoral fellow under the supervision of Professor Peter Licence. Currently, he is an Associate Professor of Organic \& Medicinal Chemistry at the Federal University of São Paulo, Brazil. He carries out studies on the immobilization of Lewis and Brønsted acids in ionic liquids or deep eutectic solvents, as unconventional reaction media and/or catalysts in multicomponent reactions applied to the synthesis of biologically active organic compounds.

\section{References}

1. Poliakoff, M.; Licence, P.; Nature 2007, 450, 810.

2. Vaccaro, L.; Eur. J. Org. Chem. 2020, 2020, 4273.

3. Varma, R. S.; ACS Sustainable Chem. Eng. 2016, 4, 5866.

4. Herrera, R. P.; Marqués-López, E.; Multicomponent Reactions: Concepts and Applications for Design and Synthesis; Wiley \& Sons, Inc.: Hoboken, NJ, 2015.

5. Gu, Y.; Green Chem. 2012, 14, 2091.

6. Fairoosa, J.; Saranya, S.; Radhika, S.; Anilkumar, G.; ChemistrySelect 2020, 5, 5180.

7. Gulati, S.; John, S. E.; Shankaraiah, N.; Beilstein J. Org. Chem. 2021, 17, 819.

8. Cioc, R. C.; Ruijter, E.; Orru, R. V. A.; Green Chem. 2014, 16, 2958.

9. Wasserscheid, P.; Welton, T.; Ionic Liquids in Synthesis; Wasserscheid, P.; Welton, T., eds.; Wiley-VCH Verlag GmbH \& Co. KGaA: Weinheim, 2002.

10. Hallett, J. P.; Welton, T.; Chem. Rev. 2011, 111, 3508.

11. Vekariya, R. L.; J. Mol. Liq. 2017, 227, 44.

12. Zhang, Q.; Zhang, S.; Deng, Y.; Green Chem. 2011, 13, 2619.

13. Steinrück, H. P.; Wasserscheid, P.; Catal. Lett. 2015, 145, 380.

14. Sawant, A. D.; Raut, D. G.; Darvatkar, N. B.; Salunkhe, M. M.; Green Chem. Lett. Rev. 2011, 4, 41.

15. Giernoth, R.; Angew. Chem., Int. Ed. 2010, 49, 2834.

16. Padvi, S. A.; Dalal, D. S.; Curr. Green Chem. 2020, 7, 105.

17. Sarma, P.; Dutta, A. K.; Borah, R.; Catal. Surv. Asia 2017, 21, 70.

18. Chiappe, C.; Rajamani, S.; Eur. J. Org. Chem. 2011, 5517.

19. Amarasekara, A. S.; Chem. Rev. 2016, 116, 6133.

20. Song, Z.; Cui, X.; Feng, T.; Zhang, Y.; Zhang, X.; He, J.; Wang, J.; J. Chem. Eng. Data 2021, 66, 1947.

21. Isambert, N.; Duque, M. M. S.; Plaquevent, J. C.; Génisson, Y.; Rodriguez, J.; Constantieux, T.; Chem. Soc. Rev. 2011, 40, 1347.

22. Parvulescu, V. I.; Hardacre, C.; Chem. Rev. 2007, 107, 2615.

23. Singh, S. K.; Savoy, A. W.; J. Mol. Liq. 2020, 297, 112038.

24. Giacalone, F.; Gruttadauria, M.; ChemCatChem 2016, 8, 664.

25. Salvo, A. M. P.; Giacalone, F.; Gruttadauria, M.; Molecules 2016, $21,1288$.

26. Xin, B.; Hao, J.; Chem. Soc. Rev. 2014, 43, 7171.

27. Li, H.; Bhadury, P. S.; Song, B.; Yang, S.; RSC Adv. 2012, 2 , 12525.

28. Pereira, M. M. A.; Curr. Org. Chem. 2012, 16, 1680.

29. Selvam, T.; MacHoke, A.; Schwieger, W.; Appl. Catal., A 2012, 445-446, 92. 
30. Skoda-Földes, R.; Molecules 2014, 19, 8840.

31. Villa, R.; Alvarez, E.; Porcar, R.; Garcia-Verdugo, E.; Luis, S. V.; Lozano, P.; Green Chem. 2019, 21, 6527.

32. Biginelli, P.; Chem. Ber. 1891, 24, 1317.

33. Kaur, R.; Chaudhary, S.; Kumar, K.; Gupta, M. K.; Rawal, R. K.; Eur. J. Med. Chem. 2017, 132, 108.

34. Nagarajaiah, H.; Mukhopadhyay, A.; Moorthy, J. N.; Tetrahedron Lett. 2016, 57, 5135.

35. Heravi, M. M.; Moradi, R.; Mohammadkhani, L.; Moradi, B.; Mol. Diversity 2018, 22, 751.

36. Heravi, M. M.; Zadsirjan, V.; Curr. Org. Chem. 2020, 24, 1331.

37. Chopda, L. V.; Dave, P. N.; ChemistrySelect 2020, 5, 5552.

38. Panda, S.; Khanna, P.; Khanna, L.; Curr. Org. Chem. 2012, 16, 507.

39. Abbasi, M.; Res. Chem. Intermed. 2016, 42, 3303.

40. Zare, A.; Nasouri, Z.; J. Mol. Liq. 2016, 216, 364.

41. Savanur, H. M.; Kalkhambkar, R. G.; Aridoss, G.; Laali, K. K.; Tetrahedron Lett. 2016, 57, 3029.

42. Wang, X.; Quan, Z.; Wang, F.; Wang, M.; Zhang, Z.; Li, Z.; Synth. Commun. 2006, 36, 451.

43. Braga, T. C.; Silva, T. F.; Maciel, T. M. S.; da Silva, E. C. D.; da Silva-Júnior, E. F.; Modolo, L. V.; Figueiredo, I. M.; Santos, J. C. C.; de Aquino, T. M.; de Fátima, Â.; New J. Chem. 2019, 43, 15187

44. Rahman, M.; Sarkar, A.; Ghosh, M.; Majee, A.; Hajra, A.; Tetrahedron Lett. 2014, 55, 235.

45. Zhou, Z. L.; Wang, P. C.; Lu, M.; Chin. Chem. Lett. 2016, 27, 226.

46. He, L.; Qin, S.; Liu, J.; Zhao, W.; Chang, T.; World J. Eng. 2020, 17, 21.

47. Alvim, H. G. O.; Pinheiro, D. L. J.; Carvalho-Silva, V. H.; Fioramonte, M.; Gozzo, F. C.; da Silva, W. A.; Amarante, G. W.; Neto, B. A. D.; J. Org. Chem. 2018, 83, 12143.

48. Fu, R.; Yang, Y.; Lai, W.; Ma, Y.; Chen, Z.; Zhou, J.; Chai, W.; Wang, Q.; Yuan, R.; Synth. Commun. 2015, 45, 467.

49. Alvim, H. G. O.; de Lima, T. B.; de Oliveira, H. C. B.; Gozzo, F. C.; de Macedo, J. L.; Abdelnur, P. V.; Silva, W. A.; Neto, B. A. D.; ACS Catal. 2013, 3, 1420.

50. Sheldon, R. A.; ACS Sustainable Chem. Eng. 2018, 6, 32.

51. Khiratkar, A. G.; Muskawar, P. N.; Bhagat, P. R.; RSC Adv. 2016, 6, 105087.

52. Rostamizadeh, S.; Nojavan, M.; Aryan, R.; Azad, M.; Catal. Lett. 2014, 144, 1772.

53. Yao, N.; Lu, M.; Liu, X. B.; Tan, J.; Hu, Y. L.; J. Mol. Liq. 2018, $262,328$.

54. Edraki, N.; Mehdipour, A. R.; Khoshneviszadeh, M.; Miri, R.; Drug Discovery Today 2009, 14, 1058.

55. Khedkar, S.; Auti, P.; Mini-Rev. Med. Chem. 2014, 14, 282.

56. Sharma, V. K.; Singh, S. K.; RSC Adv. 2017, 7, 2682.

57. Khot, S.; Auti, P. B.; Khedkar, S. A.; Mini-Rev. Med. Chem. 2021, 21, 135.
58. Malhi, D. S.; Kaur, M.; Sohal, H. S.; ChemistrySelect 2019, 4, 11321.

59. Sarma, M. D. A. S.; Ghosh, S.; Asian J. Chem. 2020, 32, 2943.

60. Hantzsch, A.; Ann. Chem. 1882, 215, 1.

61. Jassem, A. M.; Almashal, F. A. K.; Mohammed, M. Q.; Jabir, H. A. S.; SN Appl. Sci. 2020, 2, 359.

62. Liu, X.; Liu, B.; J. Chem. 2017, 2017, ID 5646908.

63. Nia, R. H.; Mamaghani, M.; Shirini, F.; Tabatabaeian, K.; Heidary, M.; Org. Prep. Proced. Int. 2014, 46, 152.

64. Jahanbin, B.; Davoodnia, A.; Behmadi, H.; Tavakoli-Hoseini, N.; Bull. Korean Chem. Soc. 2012, 33, 2140.

65. Alvim, H. G. O.; Bataglion, G. A.; Ramos, L. M.; de Oliveira, A. L.; de Oliveira, H. C. B.; Eberlin, M. N.; de Macedo, J. L.; da Silva, W. A.; Neto, B. A. D.; Tetrahedron 2014, 70, 3306.

66. Alvim, H. G. O.; Correa, J. R.; Assumpção, J. A. F.; da Silva, W. A.; Rodrigues, M. O.; de Macedo, J. L.; Fioramonte, M.; Gozzo, F. C.; Gatto, C. C.; Neto, B. A. D.; J. Org. Chem. 2018, 83,4044

67. Ren, Y. M.; Shao, J. J.; Wu, Z. C.; Xu, M. D.; Org. Prep. Proced. Int. 2014, 46, 545.

68. Ren, Y. M.; Jin, S.; Yan, H. J.; Zhang, Z.; Catalysts 2015, 5, 1649.

69. Ren, Y. M.; Yang, R. C.; Synth. Commun. 2016, 46, 1318.

70. Naeimi, H.; Nejadshafiee, V.; Islami, M. R.; Microporous Mesoporous Mater. 2016, 227, 23.

71. Mirhosseini-Eshkevari, B.; Esnaashari, M.; Ghasemzadeh, M. A.; ACS Omega 2019, 4, 10548.

72. Prajapati, S. M.; Patel, K. D.; Vekariya, R. H.; Panchal, S. N.; Patel, H. D.; RSC Adv. 2014, 4, 24463.

73. Dib, M.; Ouchetto, H.; Ouchetto, K.; Hafid, A.; Khouili, M.; Curr. Org. Synth. 2021, 18, 248.

74. Kumar, S.; Bawa, S.; Gupta, H.; Mini-Rev. Med. Chem. 2009, 9, 1648.

75. Batista, V. F.; Pinto, D. C. G. A.; Silva, A. M. S.; ACS Sustainable Chem. Eng. 2016, 4, 4064.

76. Zhang, M.; Xiong, B.; Wang, T.; Ding, Y. Q.; Wang, L.; Heterocycles 2011, 83, 2289.

77. Khaligh, N. G.; Chin. J. Catal. 2014, 35, 1858.

78. Mohammadi, K.; Shirini, F.; Yahyazadeh, A.; RSC Adv. 2015, 5,23586

79. Heravi, M. M.; Saeedi, M.; Karimi, N.; Zakeri, M.; Beheshtiha, Y. S.; Davoodnia, A.; Synth. Commun. 2010, 40, 523.

80. Zare, A.; Abi, F.; Moosavi-Zare, A. R.; Beyzavi, M. H.; Zolfigol, M. A.; J. Mol. Liq. 2013, 178, 113.

81. Ghorbani, M.; Noura, S.; Oftadeh, M.; Narimani, M.; J. Mol. Liq. 2015, 209, 224

82. Siwach, A.; Verma, P. K.; BMC Chem. 2021, 15, 12.

83. Shabalin, D. A.; Camp, J. E.; Org. Biomol. Chem. 2020, 9, 3950.

84. Noorhisham, N. A.; Amri, D.; Mohamed, A. H.; Yahaya, N.; Ahmad, N. M.; Mohamad, S.; Kamaruzaman, S.; Osman, H.; J. Mol. Liq. 2021, 326, 115340. 
85. Shaterian, H. R.; Ranjbar, M.; Azizi, K.; J. Iran. Chem. Soc. 2011, $8,1120$.

86. Zolfigol, M. A.; Khazaei, A.; Moosavi-Zare, A. R.; Zare, A.; Asgari, Z.; Khakyzadeh, V.; Hasaninejad, A.; J. Ind. Eng. Chem. 2013, 19, 721.

87. Khaligh, N. G.; Chong, K. F.; Mihankhah, T.; Titinchi, S.; Johan, M. R.; Ching, J. J.; Aust. J. Chem. 2018, 71, 566.

88. Khaligh, N. G.; Mihankhah, T.; Johan, M. R.; Synth. Commun. 2019, 49, 1334.

89. Yarie, M.; Zolfigol, M. A.; Saeidi-Rad, M.; J. Mol. Liq. 2018, 249,144

90. Abshirini, Z.; Zare, A.; Z. Naturforsch., B: J. Chem. Sci. 2018, 73, 191.

91. Rezaei, F.; Amrollahi, M. A.; Khalifeh, R.; ChemistrySelect 2020, $5,1760$.

92. Kaur, N.; SN Appl. Sci. 2019, 1, 932.

93. Mamaghani, M.; Nia, R. H.; Tavakoli, F.; Jahanshahi, P.; Curr. Org. Chem. 2018, 22, 1704.

94. Raj, V.; Lee, J.; Front. Chem. 2020, 8, 623.

95. Tashrifi, Z.; Mohammadi-Khanaposhtani, M.; Hamedifar, H.; Larijani, B.; Ansari, S.; Mahdavi, M.; Mol. Diversity 2020, 24, 1385.

96. Fang, D.; Zhang, H.-B.; Liu, Z. L.; J. Heterocycl. Chem. 2010, $47,63$.

97. Zolfigol, M. A.; Khazaei, A.; Moosavi-Zare, A. R.; Afsar, J.; Khakyzadeh, V.; Khaledian, O.; J. Chin. Chem. Soc. 2015, 62, 398.

98. Sonyanaik, B.; Sakram, B.; Kurumanna, A.; Madhu, P.; Heterocycl. Lett. 2018, 8, 375.

99. Khaligh, N. G.; Mihankhah, T.; Johan, M. R.; J. Mol. Liq. 2019, 277, 794.

100. Habibi, D.; Shamsian, A.; Nematollahi, D.; Chem. Pap. 2015, 69, 586.

101. Poupelin, J. P.; Saint-Ruf, G.; Lacroix, R.; Narcisse, G.; Foussard-Blanpin, O.; Uchida-Ernouf, G.; Eur. J. Med. Chem. 1978, 13, 67.

102. Qiao, Y.; Okazaki, T.; Ando, T.; Mizoue, K.; J. Antibiot. 1998, $51,282$.

103. Jamison, J. M.; Krabill, K.; Hatwalkar, A.; Cell Biol. Int. Rep. 1990, 74, 1075.

104. Rewcastle, G. W.; Atwell, G. J.; Zhuang, L.; Baguley, B. C.; Denny, W. A.; J. Med. Chem. 1991, 34, 217.

105. Fang, D.; Gong, K.; Liu, Z. L.; Catal. Lett. 2009, 127, 291.

106. Zolfigol, M. A.; Khakyzadeh, V.; Moosavi-Zare, A. R.; Zare, A.; Azimi, S. B.; Asgari, Z.; Hasaninejad, A.; C. R. Chim. 2012, 15,719 .

107. Ashtarian, J.; Heydari, R.; Maghsoodlou, M. T.; Yazdani-ElahAbadi, A.; Iran. J. Sci. Technol. Trans. 2020, 44, 51.

108. Sorkhi, S. E. S.; Hashemi, M. M.; Ezabadi, A.; Res. Chem. Intermed. 2020, 46, 2229.

109. Dutta, A. K.; Gogoi, P.; Saikia, S.; Borah, R.; J. Mol. Liq. 2017, $225,585$.
110. Shaterian, H. R.; Mohammadnia, M.; Moradi, F.; J. Mol. Liq. 2012, 172, 88 .

111. Shaterian, H. R.; Sedghipour, M.; Mollashahi, E.; Res. Chem. Intermed. 2014, 40, 1345.

112. Ebrahimi, J.; Mohammadi, A.; Pakjoo, V.; Bahramzade, E.; Habibi, A.; J. Chem. Sci. 2012, 124, 1013.

113. Mamaghani, M.; Nia, R. H.; Shirini, F.; Tabatabaeian, K.; Rassa, M.; Med. Chem. Res. 2015, 24, 1916.

114. Farokhian, P.; Mamaghani, M.; Mahmoodi, N. O.; Tabatabaeian, K.; J. Iran. Chem. Soc. 2018, 15, 11.

115. Zakeri, M.; Nasef, M. M.; Kargaran, T.; Ahmad, A.; AbouzariLotf, E.; Asadi, J.; Res. Chem. Intermed. 2017, 43, 717.

116. Karami, M.; Maghsoudi, M.; Merajoddin, M.; Zare, A.; Asian J. Nanosci. Mater. 2019, 2, 413.

117. Zolfigol, M. A.; Ayazi-Nasrabadi, R.; Baghery, S.; Appl. Organomet. Chem. 2016, 30, 273.

118. Sangshetti, J.; Pathan, S. K.; Patil, R.; Ansari, S. A.; Chhajed, S.; Arote, R.; Shinde, D. B.; Bioorg. Med. Chem. 2019, 27, 3979.

119. Shaterian, H. R.; Aghakhanizadeh, M.; C. R. Chim. 2012, 15, 1060.

120. Habibi, D.; Shamsian, A.; Res. Chem. Intermed. 2015, 41, 6245 .

121. Pouramiri, B.; Kermani, E. T.; Tetrahedron Lett. 2016, 57, 1006.

122. Pouramiri, B.; Far, R. G.; Zahedifar, M.; Chem. Heterocycl. Compd. 2018, 54, 1056.

123. Abedini, M.; Shirini, F.; Omran, J. M. A.; J. Mol. Liq. 2015, 212, 405.

124. Shirini, F.; Langarudi, M. S. N.; Goli-Jolodar, O.; Dyes Pigm. 2015, 123, 186.

125. Tayebee, R.; Jomei, M.; Maleki, B.; Razi, M. K.; Veisi, H.; Bakherad, M.; J. Mol. Liq. 2015, 206, 119.

126. Zare, A.; Masihpour, F.; Phosphorus, Sulfur Silicon Relat. Elem. 2016, 191, 1160.

127. Arian, F.; Keshavarz, M.; Sanaeishoar, H.; Hasanzadeh, N.; J. Mol. Struct. 2021, 1229, 129599.

128. Safaei, S.; Mohammadpoor-Baltork, I.; Khosropour, A. R.; Moghadam, M.; Tangestaninejad, S.; Mirkhani, V.; Catal. Sci. Technol. 2013, 3, 2717.

129. Hajipour, A. R.; Ghayeb, Y.; Sheikhan, N.; Ruoho, A. E.; Tetrahedron Lett. 2009, 50, 5649.

130. Luo, J.; Zhang, Q.; Monatsh. Chem. 2011, 142, 923.

131. Zare, A.; Yousofi, T.; Moosavi-Zare, A. R.; RSC Adv. 2012, 2 , 7988.

132. Kotadia, D. A.; Soni, S. S.; J. Mol. Catal. A: Chem. 2012, 353354, 44.

133. Davoodnia, A.; Heravi, M. M.; Rezaei-Daghigh, L.; TavakoliHoseini, N.; Monatsh. Chem. 2009, 140, 1499.

134. Davoodnia, A.; Heravi, M. M.; Rezaei-Daghigh, L. D.; TavakoliHoseini, N. H.; Chin. J. Chem. 2010, 28, 429.

135. Wang, Y.; Zhou, J.; Liu, K.; Dai, L.; J. Mol. Catal. A: Chem. 2013, 366, 195. 
136. Zare, A.; Moosavi-Zare, A. R.; Merajoddin, M.; Zolfigol, M. A.; Hekmat-Zadeh, T.; Hasaninejad, A.; Khazaei, A.; Mokhlesi, M.; Khakyzadeh, V.; Derakhshan-Panah, F.; Beyzavi, M. H.; Rostami, E.; Arghoon, A.; Roohandeh, R.; J. Mol. Liq. 2012, 167, 69 .

137. Boroujeni, K. P.; Taheri, S.; Seyfipour, G.; Synth. React. Inorg., Met.-Org., Nano-Met. Chem. 2014, 44, 84.

138. Irannejad-Gheshlaghchaei, N.; Zare, A.; Sajadikhah, S. S.; Banaei, A.; Res. Chem. Intermed. 2018, 44, 6253.

139. Davoodnia, A.; Attar, P.; Morsali, A.; Eshghi, H.; TavakoliHoseini, N.; Khadem, S.; Bull. Korean Chem. Soc. 2011, 32, 1873.

140. Banothu, J.; Bavantula, R.; Crooks, P. A.; Iran. J. Catal. 2013, 3,41 .

141. Zaharani, L.; Khaligh, N. G.; Mihankhah, T.; Shahnavaz, Z.; Johan, M. R.; Synth. Commun. 2020, 50, 1633.
142. Basirat, N.; Sajadikhah, S. S.; Zare, A.; Res. Chem. Intermed. 2020, 46, 3263.

143. Mohammadi, S.; Abbasi, M.; Res. Chem. Intermed. 2015, 41, 8877.

144. Vahdat, S. M.; Akbari, M.; Orient. J. Chem. 2011, 27, 1573.

145. Xiong, H.; Han, J.; Wang, J.; Lu, W.; Wang, C.; Chen, Y.; Lian, F.; Zhang, N.; Liu, Y.; Zhang, C.; Ding, H.; Jiang, H.; Lu, W.; Luo, C.; Zhou, B.; Eur. J. Med. Chem. 2018, 151, 740.

146. Kumar, M.; Sharma, K.; Arya, A. K.; Tetrahedron Lett. 2012, 53,4604 .

147. Seyyedhamzeh, M.; Shaabani, S.; Sangachin, M. H.; Shaabani, A.; Res. Chem. Intermed. 2016, 42, 2845.

Submitted: October 11, 2021

Published online: January 28, 2022 